\title{
Los acuerdos de programa en el Derecho Local Italiano*
}

\author{
Marcos Almeida Cerreda \\ Área de Derecho Administrativo de la Universidad de Santiago de Compostela
}

\begin{abstract}
Sumario: I. INTRODUCCIÓN. II. LOS ASPECTOS SUBJETIVOS DE LOS ACUERDOS DE PROGRAMA: 1) La participación de los particulares; 2) La determinación del sujeto titular del poder de promoción. III. LOS ASPECTOS OBJETIVOS DE LOS ACUERDOS DE PROGRAMA: 1) El objeto de los acuerdos de programa; 2) El contenido de los acuerdos de programa. IV. LOS ASPECTOS PROCEDIMENTALES DE LOS ACUERDOS DE PROGRAMA: 1) La formación de los acuerdos de programa: A) La fase de instrucción: a) La convocatoria de la conferencia; b) El funcionamiento de la conferencia. B) La fase de decisión: a) La determinación del contenido de los acuerdos de programa; b) La adhesión al acuerdo de programa y la suscripción del mismo. C) La fase de exteriorización: a) La aprobación de los acuerdos de programa; b) La publicación de los acuerdos de programa. 2) La eficacia de los acuerdos de programa. 3) La ejecución de los acuerdos de programa. BIBLIOGRAFÍA CITADA.
\end{abstract}

\section{INTRODUCCIÓN}

Los acuerdos de programa constituyen, en el marco del Ordenamiento jurídico italiano, un tipo especial de convenio interadministrativo que concluyen entre sí varios sujetos públicos, para coordinar su actividad, con el objetivo de simplificar y agilizar la planificación y/o ejecución de determinadas actuaciones de su competencia ${ }^{1}$.

\footnotetext{
* El presente trabajo ha sido elaborado en el marco del Proyecto BJU2003-01752 del Ministerio de Ciencia y Tecnología y del Proyecto PGIDIT03CSO20203PR de la Xunta de Galicia dirigidos ambos por el Prof. Dr. D. José Luis Carro FernÁndEZ-VALMAYoR. Las principales abreviaturas utilizadas en el mismo son las siguientes: art./arts., artículo/artículos; cfr., confere; coord., coordinador; Cons. Stato, Consiglio di Stato; Com. it., I Comuni d'Italia; Dir. amm., Diritto amministrativo; Dir. ec., Il Diritto dell'economia; D. lgs., Decreto legislativo; D.P.R., Decreto del Presidente de la República; ed., edición; Enc. dir., Enciclopedia del Diritto; fasc., fascículo; Foro amm., Il Foro administrativo; L., Ley; Leggi civ. comment., Le nuove leggi civili commentate; L. r., Ley regional; n., número; Nuova rass., Nuova rassegna di legislazione, dottrina e giurisprudenza; op. cit., opera citata; p./pp., página/páginas; p. ej., por ejemplo; par., parte; Quad. reg., Quaderni regionali; Regioni, Le Regioni; Riv. amm., Rivista amministrativa della Repubiblica Italiana; Riv. it. dir. pubbl. com, Rivista italiana di Diritto pubblico comunitario; Riv. giur. mezz., Rivista giuridica del Mezzogiorno; Riv. giur. urb., Rivista giuridica di urbanistica; Riv. trim. appalti, Rivista trimestrale degli appalti; S., Sentencia; s./ss., siguiente/siguientes; Sez., Sezione; TAR, Tribunale Anuninistrativo Regionale; tb., también; Trib. amm. reg., I Tribunali Amministrativi Regionali; TUEL, Testo Unico delle Leggi sull'Ordinamento degli Enti Locali; vid., vide; vol., volumen.

${ }^{1}$ En Italia la doctrina mayoritaria considera que los acuerdos de programa son una especie del género convenio interadministrativo, en este sentido, vid., entre otros muchos: E. STICCHI DAMIANI, Atti-
} 


\section{Desde su introducción en el Derecho administrativo italiano, este ha conocido un espectacular desarrollo que todavía no ha alcanzado negocio jurídico su cenit ${ }^{2}$. Día a día es empleado con mayor frecuencia por las}

vità amministrativa consensuale e accordi di programma, Giuffrè, Milano, 1992, p. 14; R. FERRARA, Gli accordi di programma. Potere, poteri pubblici e modelli dell'amministrazione concertata, Cedam, Padova, 1993, pp. 63, 101 y 140; G. D. COMPORTI, Il coordinamento infrastrutturale. Tecniche e garanzie, Giuffrè, Milano, 1996, pp. 321-323; S. CivitARESE MatTeuCCI, «Accordo di programma», Enc. dir., Giuffrè, Milano, 1999, pp. 12-14 y 23; G. FALCON, «Gli accordi tra amministrazioni e tra amministrazioni e privato», La semplificazione amministrativa, a cargo de L. VANDELLI y G. GARDINI, Rimini, Maggioli, 1999, p. 164; C. Di MARCO, «I rapporti Stato-Regioni-Enti locali nel sistema dell'amministrazione pattizia», Regioni, n. ${ }^{\circ} 3$, 1999, p. 478; R. DAMONTE, Atti, accordi, convenzioni nella giustizia amministrativa, Cedam, Padova, 2002, pp. 159 y 249. Hay que señalar que también la jurisprudencia comparte esta opinión, vid., p. ej., la S. Cons. Stato, Sez. VI, de 5 de enero de 2001, n. ${ }^{\circ} 25$. Sobre la teoría general de los convenios interadministrativos en el Ordenamiento jurídico italiano, aplicable a los acuerdos de programa en la medida en que éstos son un tipo particular de aquellos, vid.: M. ALMEIDA CERREDA, Los copnvenios interadministrativos en el Derecho italiano», Revista de Administración Pública, nº 163 pp. 389 ss.

${ }^{2}$ El origen de estos acuerdos de programa se halla, posiblemente, en la transposición y reelaboración en el Derecho italiano de los denominados «contratos de programa» regulados por los Reglamentos de la Comunidad Económica Europea n. ${ }^{\circ}$ 1787/84, de 19 de junio, y n ${ }^{\circ} .2088 / 85$, de 23 de julio, que se refieren, respectivamente, al Fondo Europeo de Desarrollo Regional y a los Programas Integrados Mediterráneos (vid. S. CivitAReSE MATTEUCCI, «Accordo di programma», op. cit., p. 11, nota 10). Estos contratos de programa son un tipo de acuerdo entre sujetos públicos, cuyo objeto es la previsión de una serie de medidas de coordinación, dirigidas a organizar la participación de dichos sujetos en la ejecución de una actuación administrativa previamente programada (vid., sobre este instrumento propio del Derecho comunitario: C. D'ORTA, «I programmi integrati mediterranei in Italia: disciplina e prima attuazione», Riv. giur mezz., $\mathrm{n}^{\circ}$. 1, 1988, p. 101; G.F. CARTEI, «Gli accordi di programma nel diritto comunitario e nazionale», Riv. it. dir pubbl. Com., no . 1, 1991, p. 61; E. STICCHI DAMIÁN, «Gli accordi amministrativi», La contrattualizzazione dell'azione amministrativa, a cargo de C. Amirante, Giappichelli, Torino, 1993, pp. 154-156). Algunos autores opinan que, a su vez, es muy probable que estos contratos de programa se hayan inspirado en los contrats plan fraceses (vid., en este sentido, A. FIORITTO, «Accordi di programma e contrats plan: somiglianze e divergenze», Riv. Giur. mezz., n. ${ }^{\circ} 3$, 1989, p. 873; G.D. COMPORTI, Il coordinamento infrastrutturale, op. cit., p. 248). Hay que destacar que el proceso de absorción por el Derecho italiano de la citada figura jurídica no se llevó a cabo, como sería deseable, mediante la aprobación de una única Ley destinada a regularla de modo general y uniforme. Por el contrario, la adopción de los acuerdos de programa se produjo como consecuencia de la aprobación de sucesivas leyes sectoriales, cada una de las cuales diseñó una concreta regulación que presentaba diferencias, más o menos marcadas -sea en el objeto, sea en el procedimiento, sea en los efectos- que respondían a las particulares necesidades que trataban de satisfacer cada una de dichas normas. En este sentido, conviene señalar que muchas de tales normas tenían en común que fueron promulgadas para hacer frente a situaciones caracterizadas por una cierta emergencia -inicialmente, los acuerdos de programa fueron empleados como instrumento para la programación o la realización acelerada de intervenciones infraestructurales, como la construcción de obras ferroviarias, o como instrumento para la intervención extraordinaria en el Mediodía (vid., el apartado 3 del art. 25 de la L. n. ${ }^{\circ}$ 210/1985, de 17 de mayo, Istitutiva dell'Ente Ferrovie dello Stato, y el art. 7 de la L. n. 64/1986, de 1 de marzo, Disciplina organica dell'intervento straordinario nel Mezzogiorno)-. Este tortuoso modo en el que se produjo la inserción de los acuerdos de programa en el Ordenamiento italiano ocasionó una acentuada y perniciosa falta de uniformidad de esta figura jurídica (vid. E. STICCHI DAMIANI, Attività amministrativa consensuale e accordi di programma, op. cit., p. 6), que aún se mantiene en la actualidad, si bien la entrada en vigor de la L. n. ${ }^{\circ}$ 142/1990, de 8 de junio, Ordinamento delle autonomie locali, miti- 
Administraciones públicas, sobre todo por los Entes locales, para llevar a cabo de modo conjunto todo tipo de actuaciones. A juicio de la doctrina italiana, el origen de este auge radica en que los acuerdos de programa se han demostrado extremadamente útiles para lograr la coordinación de los sujetos públicos que los suscriben, con las ventajas que ello conlleva, las cuales van desde la simplificación y agilización de la actividad administrativa, a través de la eliminación de los tiempos muertos que, habitualmente, caracterizan la programación y ejecución de actuaciones que implican a varios niveles de gobierno, hasta el evitar la repetición de actuaciones por parte dichos sujetos, permitiéndoles, en consecuencia, una considerable reducción de gastos ${ }^{3}$. Dado este éxito de los acuerdos de programa en el Ordenamiento italiano, y tendiendo en cuenta que dicho Ordenamiento tiene notables similitudes con el español, me ha parecido interesante realizar el presente estudio sobre este instrumento jurídico, con el anhelo de poder facilitar la introducción en nuestro acervo jurídico de alguna de las características de los acuerdos de programa que les han permitido desarrollarse con tal fuerza, de modo que la regulación de los instrumentos de cooperación convencional de los Entes locales que actualmente existen en el Derecho Local español se vea completada y mejorada. Para alcanzar este objetivo, en el presente estudio, partiendo de la regulación contenida en el D. lgs. n. ${ }^{\circ}$ 267/2000, de 18 de agosto, Testo Unico delle Leggi sull'Ordinamento degli Enti Locali (en adelante, $\mathrm{TUEL})^{4}$, se lleva a cabo una descripción crítica de la configuración y fun-

gó dicha heterogeneidad, con la introducción de un modelo general de referencia, aunque éste sólo era aplicable a los acuerdos de programa suscritos por los Entes locales.

${ }^{3}$ Cfr. F. CAIFFA, «Le nuove frontiere dell'attività amministrativa consensuale: gli accordi di programma», Nuova rass., n. ${ }^{\circ} 3-4,1993$, p. 233; N. BASSI, «Commento all'articolo 34», Testo Unico degli Enti Locali, coord. V. Italia, vol. I, tomo I, Giuffrè, Milano, 2000. p. 397; G. PERICU, «L'attivitá consensuale della amministrazione pubblica», Diritto amministrativo, a cargo de L. MAzzARoLLI y otros, vol. II, Monduzzi Editore, Bologna, $3^{\text {a }}$ ed., 2001, p. 1686.

${ }^{4}$ La regulación de los acuerdos de programa se halla contenida en el artículo 34 de este texto, cuya redacción no introduce ninguna innovación respecto a la normativa preexistente. Se trata de una mera repetición del art. 27 de la L. n. ${ }^{\circ}$ 142/1990, de 8 de junio, Ordinamento delle autonomie locali, con las modificaciones que en el mismo introdujeron los apartados 8 y 9 del art. 17 de la L. n. ${ }^{\circ}$ 127/1997, de 15 de mayo, denominada Bassanini bis. El tenor literal de este artículo 34 es el siguiente:

«1. Per la definizione e l'attuazione di opere, di interventi o di programmi di intervento che richiedono, per la loro completa realizzazione, l'azione integrata e coordinata di comuni, di province e regioni, di amministrazioni statali e di altri soggetti pubblici, o comunque di due o più tra i soggetti predetti, il presidente della regione o il presidente della provincia o il sindaco, in relazione alla competenza primaria o prevalente sull'opera o sugli interventi o sui programmi di intervento, promuove la conclusione di un accordo di programma, anche su richiesta di uno o più dei soggetti interessati, per assicurare il coordinamento delle azioni e per determinarne i tempi, le modalità, il finanziamento ed ogni altro connesso adempimento. 
cionamiento de los acuerdos de programa que no es (ni podría serlo, dados los insoslayables límites de extensión que tiene todo artículo) exhaustiva. Esencialmente, dicha descripción se ciñe a mostrar quiénes pueden intervenir en los acuerdos de programa, para qué se suscriben, cómo se elaboran y en qué modo se ejecutan.

"2. L'accordo può prevedere altresì procedimenti di arbitrato, nonché interventi surrogatori di eventuali inadempienze dei soggetti partecipanti.

»3. Per verificare la possibilità di concordare l'accordo di programma, il presidente della regione o il presidente della provincia o il sindaco convoca una conferenza tra i rappresentanti di tutte le amministrazioni interessate.

»4. L'aecordo, consistente nel consenso unanime del presidente della regione, del presidente della provincia, dei sindaci e delle altre amministrazioni interessate, è approvato con atto formale del presidente della regione o del presidente della provincia o del sindaco ed è pubblicato nel bollettino ufficiale della regione. L'accordo, qualora adottato con decreto del presidente della regione, produce gli effetti dell'intesa di cui all'articolo 81 del decreto del Presidente della Repubblica 24 luglio $1977, \mathrm{n}^{\circ}{ }^{\circ} 616$, determinando le eventuali e conseguenti variazioni degli strumenti urbanistici e sostituendo le concessioni edilizie, sempre che vi sia l'assenso del comune interessato.

»5. Ove l'acoordo comporti variazione degli strumenti urbanistici, l'adesione del sindaco allo stesso deve essere ratificata dal consiglio comunale entro trenta giorni a pena di decadenza.

»6. Per l'approvazione di progetti di opere pubbliche comprese nei programmi dell'araministrazione e per le quali siano immediatamente utilizzabili i relativi finanziamenti si procede a norma dei precedenti commi. L'approvazione dell'accordo di programma comporta la dichiarazione di pubblica utilità, indifferibilità ed urgenza delle medesime opere; tale dichiarazione cessa di avere efficacia se le opere non hanno ayuto inizio entro tre anni.

»7. La vigilanza sull'esecuzione dell'accordo di programma e gli eventuali interventi sostitutivi sono svolti da un collegio presieduto dal presidente della regione o dal presidente della provincia o dal sindaco e composto da rappresentanti degli enti locali interessati, nonché dal commissario del Governo nella regione o dal prefetto nella provincia interessata se all'accordo partecipano amministrazioni statali o enti pubblici nazionali.

»8. Allorché l'Intervento o il programma di intervento comporti il concorso di due o più regioni finitime, la conclusione dell'acoordo di programma è promossa dalla Presidenza del Consiglio dei Ministri, a cui spetta convocare la conferenza di cui al conuna 3. Il collegio di vigilanza di cui al comma 7 è in tal caso presieduto da un rappresentante della Presidenza del Consiglio dei Ministri ed è composto dai rappresentanti di tutte le regioni che hanno partecipato all'accordo. La Presidenza del Consiglio dei Ministri esercita le fimioni attribuite dal conmma 7 al commissario del Governo ed al prefetto».

Este precepto junto con las Leyes regionales sobre esta materia (como, p. ej.: L. r. Puglia n. ${ }^{\circ} 4 / 1995$, de 20 de febrero, Disciplina delle procedure per gli accordi di programma; L. r. Toscana n. 76/1996, de 3 de septiembre, Disciplina degli accordi di programma e delle conferenze dei servizi; L. r. Molise n. ${ }^{\circ}$ 17/1999, de 11 de junio, Disciplina del procedimento per l'accordo di programma; L. r. Lombardia n. 2/2003, de 14 de marzo, Programmazione negoziata regionale) y los Estatutos de los Entes locales constituyen el marco normativo dentro del cual han de moverse los acuerdos de programa de los Entes locales en Italia. 


\section{LOS ASPECTOS SUBJETIVOS DE LOS ACUERDOS DE PROGRAMA}

La forma en la que el artículo 34 TUEL disciplina la vertiente subjetiva de los acuerdos de programa plantea dos problemas interpretativos que los operadores jurídicos han de resolver a la hora de aplicarlo. El primero, que reviste carácter general, en cuanto que su solución necesariamente será idéntica para todos los acuerdos de programa, con abstracción de las particulares circunstancias en las que se desenvuelva cada uno de ellos, consiste en determinar si en estos convenios pueden intervenir solamente sujetos públicos o, por el contrario, también pueden participar en ellos los particulares. El segundo problema, que, por el contrario, tiene carácter relativo, en la medida en que su solución variará en cada caso, dependiendo de las condiciones en las que se desarrolla cada concreto acuerdo de programa, consiste en precisar, aplicando las vagas directrices que establece el artículo 34 TUEL, a cuál de los sujetos que están dispuestos a tomar parte en un determinado acuerdo de programa le corresponde el poder de promover dicho acuerdo ${ }^{5}$.

\section{1) La participación de los particulares en los acuerdos de programa}

Es conveniente comenzar el estudio de este problema subrayando tanto el interés de los particulares en participar en los acuerdos de programa, como el interés de las Administraciones públicas en que los particulares intervengan en dichos acuerdos ${ }^{6}$. Desde el punto de vista de las Administraciones públicas, el ensanchamiento de la base subjetiva de los acuerdos de programa permite maximizar el cumplimiento del principio de eficiencia de la acción administrativa, ya que no cabe duda de que una de las vías para cumplir la exigencia de economía de medios en la realización de los objetivos públicos, que esencialmente demanda dicho principio, consiste

\footnotetext{
${ }^{5}$ Este poder se traduce, como se verá, en la responsabilidad de iniciar e impulsar el desarrollo del proceso que conduce a la suscripción de un determinado acuerdo de programa, procurando que dicho proceso culmine con éxito.

${ }^{6}$ En esta línea, Alberto PrEDIERI destaca: «L'accordo fornisce uno strumento utile all'Amministrazione nei confronti dei privati e, anche, ai privati che hamo a disposizione uno «sportello unico», facilita accordi per operazioni complesse (per ipotesi teoriche, costruzione di un porto o di un aeroporto, tanto privato che pubblico, o di un centro intermodale o di rete di viabilità) che possono richiedere interventi privati sia per l'esecuzione e l'attuazione, anche finanziaria, sia per la discussione informale e preliminare di progetti» («Gli aecordi di programa», Quad reg., n. ${ }^{\circ} 4,1991$, p. 972).
} 
en llevar a cabo la planificación de las actuaciones públicas, teniendo en cuenta la globalidad de los intereses implicados en ellas ${ }^{7}$. A su vez, desde el punto de vista de los particulares, es imprescindible su intervención en los acuerdos de programa, puesto que, si no participan en ellos, como consecuencia del vínculo que para los sujetos públicos nace de dicho acuerdo a los citados particulares no les quedaría más remedio que soportar, sin ni siquiera poder opinar, la ponderación de los intereses afectados por los acuerdos de programa que los sujetos públicos hayan efectuado, ponderación que, en ocasiones, puede minusvalorar considerablemente los intereses de los particulares ${ }^{8}$.

La solución más simple y directa que se puede diseñar para conseguir atender tanto los citados intereses de las Administraciones públicas, como los de los particulares, consiste en atribuir a estos últimos la condición de partes en los acuerdos de programa. Así lo han hecho algunas Leyes regionales y determinadas Leyes estatales especiales ${ }^{9}$. Sin embargo, ésta no es la solución que, con carácter general, se ha adoptado en el Ordenamiento jurídico italiano, ya que, dado el tenor literal del art. 34 TUEL, que se refiere exclusivamente a Entes públicos, de acuerdo con la doctrina mayoritaria, no se puede reconocer a los particulares la condición de parte en los acuerdos de programa. Para ello, es necesario que exista una previsión legal expresa, como la que se establece en las normas mencionadas ${ }^{10}$.

\footnotetext{
${ }^{7}$ No obstante, Vincenzo BALDINI pone de relieve que no todo son ventajas. Según este autor, la ampliación del ámbito subjetivo de los acuerdos de programa puede provocar un efecto no coherente con la ratio de los mismos, ya que puede resultar penalizada la eficiencia administrativa, debido a que, dada la pluralidad de intereses implicados, se podrían prolongar sensiblemente los tiempos de decisión administrativa («L'accordo di programma nella legislazione regionale», Regioni, n. ${ }^{\circ} 6,1992$, pp. 1649-1650, nota 102).

8 Vid. A. MAVELli, «Azione e organizzazione nell'accordo di programma», Procedimenti e accordi nell'amministrazione locale. Atti del XLII Convegno di studi di scienza dell'amministrazione, Tremezzo, 19-21 settembre 1996, Giuffrè, Milano, 1997, pp. 426-427.

${ }^{9}$ Como ejemplo se pueden citar: la L. r. Lombardia n. ${ }^{\circ} 55 / 1986$, de 25 de noviembre, Modifiche ed integrazioni alla Legge Regionale 31 marzo $1978, n{ }^{\circ} 34$, concernente "Norme sulle procedure della programmazione, sul bilancio e sulla contabilità della Regione»; la L. r. Sardegna n. ${ }^{\circ} 45 / 1989$, de 22 de dicembre, Norme per l'uso e la tutela del territorio regionale; la L. r. Veneto n. ${ }^{\circ} 40 / 1990$, de 30 de abril, Procedure della programmazione; la L. r. Friuli-Venezia Giulia n. ${ }^{\circ} 7 / 2000$, de 20 de marzo, Testo unico delle norme in materia di procedimento amministrativo e diritto di acceso; la L. n. ${ }^{\circ}$ 166/2002, de 1 de agosto, Disposizioni in materia di infrastrutture e trasporti.

10 Vid., entre otros muchos: G. SCIULlo, «Sintonie e dissonanze fra le L. 8 giugno $1990 \mathrm{n}^{\circ} .142$ e 7 agosto 1990 n..$^{\circ}$ 241: riflessi sull'autonomia locale», Foro amm., 1990, p. 2201, nota 11; G. ManFREDI, «Modelli contrattuali dell'azione amministrativa: l'accordo di programa», Regioni, n. ${ }^{\circ} 2$, 1992, p. 363; R. FERRARA, Gli accordi di programma, op. cit., p. 105; F. CAIAFFA, «Le nuove frontiere dell'attività amministrativa consensuale: gli accordi di programa», Nuova rass., n. ${ }^{\circ} 3-4,1993$, p. 234; F. PAOLUCCI, «I soggetti degli accordi di programma», Livelli e contenuti della pianificazione territoriale, a cargo de E. Ferrari, N. Santa y A. Tigano, Giuffrè, Milano, 2001, p. 299.
} 
Independientemente de si existe o no dicha previsión legal, y, por tanto, de si se puede reconocer a los particulares la condición de parte en determinados acuerdos de programa, cabe plantearse si ésta es, técnicamente, la solución idónea para articular su participación en los mismos. En este sentido, hay que tener en cuenta que el empleo de un acuerdo de programa para regular las relaciones entre sujetos públicos y privados, por una parte, supone desvirtuar este instrumento jurídico, pues de facto se produce su transformación en un acto que, si bien es idéntico bajo el aspecto formal a un acuerdo de programa, sería más bien asimilable a un contrato de programa ${ }^{11}$; por otra parte, implica situar a los sujetos públicos y privados en un plano de absoluta paridad, en el cual ambos gozan de iguales derechos y obligaciones. Esto último puede generar no pocas disonancias; así, por ejemplo, cuando, durante la ejecución de los acuerdos de programa, surja la exigencia de ejercitar los poderes de sustitución eventualmente previstos, si la parte incumplidora es un sujeto público, no sería posible negar al sujeto privado el derecho de ejercitar dichos poderes ${ }^{12}$. Por todo ello, parece más adecuado sostener que a los particulares ha de reconocérseles la posibilidad de participar en la elaboración y ejecución de los acuerdos de programa, no directamente, como partes, sino de modo indirecto, como interesados.

Esta forma transversal de intervención de los particulares se concreta en varias posibilidades de actuación de los mismos en tres momentos diferentes de la vida de los acuerdos de programa. En primer lugar, siempre que lo deseen, los particulares pueden intentar estimular la actuación de los sujetos públicos habilitados para proceder a la promoción y conclusión de los acuerdos de programa, con el objetivo de lograr que se inicie la fase

\footnotetext{
${ }^{11}$ Cfr. V. BALDINI; «L'accordo di programma nella legislazione regionale» op. cit., pp. 1626-1627. Hay que diferenciar claramente los acuerdos de programa de los contratos de programa (que, por su parte, tampoco se deben confundir con la homónima figura existente en el Derecho comunitario, a la que se ha hecho referencia en la nota número 2). Así, como señala la doctrina, mientras que el acuerdo de programa constituye, como se verá, en mayor o menor medida, un acto de planificación, el contrato de programa se manifiesta, casi siempre, como un instrumento ejecutivo de las previsiones contenidas en un acto planificador preexistente. Sin embargo, los autores destacan que no es ésta la diferencia más relevante entre ambas figuras. Las verdaderas divergencias entre ambas figuras se hallan tanto en los sujetos legitimados para estipular cada uno de estos tipos de negocios como en los fines que se persiguen con su suscripción. Así, mientras que los acuerdos de programa se concluyen, generalmente, entre Entes y Administraciones públicas con el fin de garantizar orden y coherencia a la acción administrativa, los contratos de programa su vez, se celebran entre Administraciones y sujetos privados, habitualmente empresas, para dirigir la actividad económica de éstos hacia objetivos que responden a intereses públicos, a cambio de la prestación de utilidades públicas de distintas clases (incentivos financieros, realización de infraestructuras especificas, etc...).

12 Cfr. L. ChIEFFI, «Gli strumenti per il coordinamento della ricerca scientifica: l'accordo di programma introdotto dalla lene n. ${ }^{\circ} 168$ del 1989», Riv. Giur. Mezz., n. ${ }^{\circ} 3,1992$, p. 727.
} 
de elaboración de un determinado acuerdo de programa con un cierto contenido ${ }^{13}$. En segundo lugar, en la fase de elaboración de los acuerdos de programa, pueden intervenir en el procedimiento, a través del cual se individúa el objeto de cada concreto acuerdo, mediante un trámite de audiencia que debe estar abierto a todos los sujetos potencialmente interesados en sus efectos ${ }^{14}$. Por último, en tercer lugar, en la fase de ejecución, una vez que los acuerdos de programa han sido concluidos y siempre que en los mismos se prevea, las Administraciones públicas participantes en ellos pueden suscribir con los particulares sucesivos convenios destinados a disciplinar el desarrollo de las actividades contempladas en los citados acuerdos de programa ${ }^{15}$.

${ }^{13}$ Cfr. G. Di GaSPARE, «L'acoordo di programma: strutture, efficacia giuridica e problemi di gestioni», Regioni, n. ${ }^{\circ} 2$, 1988. p. 287; R. TOMEI, «Strumenti di coordinamento amministrativo per lo sviluppo del Mezzogiorno: 1'acoordo di programa» Trib. Amm. reg., n. ${ }^{\circ} 7-8,1990$, parte II, p. 258; R. FERRARA, Gli accordi di programa, op. cit., p. 105; G.D. COMPORTI, Il coordinamento infrastrutturale, op. cit., p. 270.

${ }^{14}$ Cfr. G. TurCo Liveri, «Gli accordi di programma», Com. it., n. ${ }^{\circ} 9,1995$, p. 1262. Hay que señalar que es crucial que tanto el Legislador como las Administraciones públicas promuevan la participación de los particulares en esta fase de definición de los contenidos de los acuerdos de programa, pues éste es el único modo de lograr que las Administraciones actuantes tengan una visión completa de todos los intereses afectados por la aprobación de un acuerdo de programa, sean éstos públicos o privados, que les permita llevar a cabo una adecuada ponderación de los mismos. En determinados ámbitos, como p. ej., el urbanismo, ciertas Leyes regionales han articulado laudables soluciones para posibilitar una adecuada participación de los particulares en la formación de estos instrumentos convencionales, vid., p. ej., el art. 36 de la L. r. Toscana n. ${ }^{\circ}$ 5/1995, de 15 de enero, Norme per il governo del territorio; el art. 2 de la L. r. Puglia n. ${ }^{\circ} 4 / 1995$, de 20 de febrero, Disciplina delle procedure per gli accordi di programma; el art. 58 de la L. r. Liguria n. ${ }^{\circ} 36 / 1997$, de 4 de septiembre, Legge urbanistica regionale; y el art. 40 de la L. r. Emilia-Romagna n. ${ }^{\circ} 20 / 2000$, de 24 de marzo, Disciplina generale sulla tutela e l'uso del territorio.

${ }^{15}$ Cfr. L. CHIEFFI, «Gli strumenti per il coordinamento della ricerca scientifica: l'accordo di programma introdotto dalla legge n. 168 del 1989» op. cit., p. 727, n. ${ }^{\circ} 46$. Esta posibilidad ha sido valorada positivamente por el Dipartimento per la Funzione Pubblica en un informe de 1994, en el que, en concreto, se dice: «[In particolare, si può prevedere che] l'accordo stabilisca e definisca il rapporto con il soggetto privato, attraverso la previsione di forme societarie miste o convenzioni o «contratti di programma, che vengono conclusi «a valle" dell'accordo, per la realizzazione degli interventi previsti. Ciò favorirebbe l'attuazione dell'accordo di programma, evitando una eccessiva dispersione dei soggetti e una moltiplicazione delle procedure. In particolare, al meno quattro sono i vantaggi, che ne discenderebbero: maggiore possibilità per le pubbliche Amministrazioni di confrontare e valutare la globalità degli interessi coinvolti, pubblici e privati, in modo da operare scelte razionali in rapporto ai fini prefissati; migliore coordinamento delle azioni di competenza dei diversi soggetti e conseguente semplificazione delle procedure amministrative; trasformazione della pubblica Amministrazione in un interlocutore forte e unitario con il quale si possa confrontare il soggetto privato; maggiore interesse da parte del soggetto privato a realizzare investimenti di concerto con l'Amministrazione, in un quadro di maggiore certezza delle decisione, nonché dei tempi e dei modi della loro realizzazione; possibile soluzione in via simultanea del problema del soggetto promotore, del soggetto attuatore, del soggetto tecnico e di quello finanziario» (PRESIDENZA DEL CONSIGLIO DEI MIN ISTRI-DIPARTIMENTO DELLA FUNZIONE PUBBLICA, «Gli accerdi di programa», La semplificazione dei procedimenti di spesa per gli investimenti pubblici, Istituto Poligrafico e Zecca dello 


\section{2) La determinación del sujeto titular del poder de promoción}

\section{De acuerdo con el apartado 1 del artículo 34 TUEL, de entre todos los} sujetos que están legitimados para participar en un acuerdo de programa, corresponde a uno solo de ellos el poder de promover su conclusión ${ }^{16}$. Este poder consiste en la facultad de iniciar e impulsar el proceso encaminando a la suscripción de dicho acuerdo procurando su logro ${ }^{17}$. Hay que destacar, como lo hacen la doctrina y la jurisprudencia, que la correcta individuación del sujeto al que le pertenece el poder de promoción es fundamental, pues de ella depende la validez del acuerdo mismo, ya que, en el caso de que dicha individuación se haga de modo incorrecto, el acuerdo resultará viciado por incompetencia ${ }^{18}$.

Stato, Roma, 1994, p. 78). En este sentido, por ejemplo, el apartado 3 del art. 3 de la L r. Toscana, n. ${ }^{\circ} 76 / 1996$, de 3 de septiembre, Disciplina degli accordi di programma e delle conferenze dei servizi, dispone: "Qualora l'accordo di programina di cui al comina 1, lett. b) preveda opere, interventi, o programmi di intervento per la cui realizzazione sia necessario anche l'intervento di soggetti privati, l'accordo di programma ne da atto e può prevedere la contestuale o successiva sottoscrizione di accordi, ai sensi dell'art. 11 della L. n. ${ }^{\circ} 241 / 1991$, tra le amministrazioni pubbliche partecipanti all'aceordo di programma e competenti alla emanazione di atti autorizzativi, concessori o di assenso comunque denominati ed i soggetti privati interessati. Tali accordi, che possono essere sostitutivi degli atti autorizzativi, concessori o di assenso comunque denominati, ovvero determinare il contenuto discrezionale, stabiliscono altresì gli impegni assunti dai soggetti privati, tra i quali, in particolare, il rispetto dei contratti collettivi di lavoro e delle norme sulla sicurezza nei luoghi di lavoro, con la previsione di idonee garanzie per i casi di inadempimento».

${ }^{16}$ Literalmente este apartado dice: «[...] il presidente de la regione o il presidente de la provincia o il sindaco, in relazione a la competenza primaria o prevalente sull'opera o sugli interventi o sui programmi di intervento, promuove la conclusione di un accordo di programma $[\ldots]$...

${ }^{17}$ Hay que distinguir lo que es el poder de promoción que, efectivamente, va a corresponder a uno sólo de los sujetos interesados en la celebración de un acuerdo de programa, de la facultad de propuesta que corresponde a cualquiera de ellos. Así lo hace la S. TAR Marche, de 20 de diciembre de 2001, n. ${ }^{\circ}$ 554, cuando explica: «La disposizione [l'articolo $27 \mathrm{~L} \mathrm{n.}{ }^{\circ}$ 142/1990] assegna «ai soggetti interessati" il potere de iniziativa di avvalersi della procedura dell'accordo di programma, mentre al Sindaco attribuisce il compito di promuovere la sua conclusione, nel senso letterale di "provocare" o "portare a compimento"». Con respecto a la facultad de propuesta, Guido Corso se muestra partidario de que ésta no se concentre, sino que se reconozca a la totalidad de los sujetos, públicos y privados, interesados, ya que, en su opinión, sólo incentivando el input es pensable que este instrumento de simplificación y coordinación de la acción administrativa entre en movimiento vigorosamente («Gli accordi di programa», Riv. giur. mezz., n. ${ }^{\circ} 1$ 1, 1988, p. 16). En el mismo sentido, aunque refiriéndose al poder de promoción del acuerdo de programa, Giuseppe MANFREDI critica la concentración de dicho poder en uno solo de los potenciales participantes en el acuerdo. Según este investigador, impedir que este poder pueda ser ejercido por cualquiera de los entes interesados conlleva un grave riesgo de impasse («Commento all’ art. 15 L. 7 agosto 1990 n. ${ }^{\circ} 241 »$, Leggi civ. comment., $\left.\mathrm{n}^{\circ} 1,1995, \mathrm{p} .89\right)$.

${ }^{18}$ Cfr. S. TAR Lazio, Sez. I, de 20 de enero de 1995, n. 62; S. TAR Toscana, Sez. I. de 10 de junio de 1998, n. ${ }^{\circ}$ 346; S. TAR Marche, de 5 de noviembre de 1999, n. ${ }^{0} 1264$. 
El Legislador italiano regula, en el citado apartado 1 del artículo 34 TUEL, cómo ha de llevarse a cabo la determinación del sujeto promotor. En primer lugar, el mismo Legislador selecciona directamente, de entre los sujetos que pueden tomar parte en los acuerdos de programa, aquellos que podrán ejercer el poder de promoción; $y$, en segundo lugar, establece los criterios que los operadores jurídicos han de emplear para individuar dentro de tal elenco el sujeto que, efectivamente, en cada caso, puede ejercer dicho poder. Así, el TUEL señala como posibles titulares del poder de promoción al Alcalde, al Presidente de la Provincia y al Presidente de la Región, con lo que excluye de la titularidad de dicho poder a las Administraciones estatales y a los demás sujetos públicos ${ }^{19}$. En segundo lugar, el Legislador tras haber establecido quiénes son los sujetos que pueden ejercer del poder de promoción, procede a establecer una regla para determinar, en cada caso concreto, cuál es el sujeto al que corresponde dicho poder $^{20}$. De acuerdo con el tenor literal del apartado 1 del art. 34 TUEL, la actividad de impulso respecto a la conclusión del acuerdo será desarrollada por el Presidente de la Región o de la Provincia o por el Alcalde de acuerdo con la competencia primaria o prevalente sobre el objeto del acuerdo de programa de que se trate. Dos son, pues, los criterios que ofrece la citada norma al operador jurídico para que éste individúe la autoridad a la que ha de corresponder el poder de promoción: la primariedad y la prevalencia. El primer criterio, la primariedad, es una regla de orden jurídico. Se basa en la determinación, a través del análisis de los parámetros de distribución de la potestad previstos por el Ordenamiento jurídico,

\footnotetext{
${ }^{19}$ Las Administraciones estatales, aunque pueden tomar parte en los acuerdos de programa, no tendrán, en ningún caso, el poder de promoción del acuerdo de programa. En los supuestos en que la competencia para la realización de una actividad administrativa, objeto de un acuerdo de programa, radique en el Estado, o en una Administración nacional, parece razonable sostener que. el procedimiento de formación del acuerdo será activado por la Región (vid. B. CARAVITA, «Gli accordi di programma», Azienditalia, n. ${ }^{\circ}$ 9, 1990, pp. 38 ss.). En este sentido Guido GRECo ha afirmado: «[...] per le opere di competenza statale l'iniziativa all'accordo di programma è dissociata dalla competenza. E deve essere individuata [...] in capo al presidente della regione, almeno tutte le volte in cui la localizzazione dell'opera o del tracciato (si pensi ad una linea ferroviaria) è difforme dagli strumenti urbanistici dei singoli comuni interessati: ciò in relazione all'efficacia dell'accordo di programma, che, ai sensi del comma 4 del medesimo art. 27, produce gli effetti dell'intesa di cui all'art. 81 del d. P. R. 616 del 1977 solo qualora sia adottato con decreto del presidente de la regione» («Commento all'art. 27», Le autonomie locali, coordinado por V. ITALIA y M. BASSANI, tomo I, Qiuffrè, Milano, 1990, p. 391). Con esta exclusión, la doctrina entiende que el Legislador pretende garantizar el pleno respeto de la autonomía de los Entes locales, asegurando que, desde el inicio, las relaciones convencionales entre los distintos niveles de gobierno se desenvuelvan por vías de efectiva paridad.

${ }^{20}$ Como señala Guido Greco, el Legislador ha recurrido para este fin a una disposición elástica, que permite la adaptación a las más variadas hipótesis de participación en el acuerdo de programa, pero que, en contrapartida, comporta en numerosos casos notables incertidumbres y dificultades de aplicación («Commento all’ at 27», op. cit., p. 390).
} 
de la autoridad administrativa que tiene atribuida una competencia activa exclusiva en relación con el objeto del acuerdo de programa ${ }^{21}$. El segundo criterio, la prevalencia, se presenta como una pauta de naturaleza empírica y de carácter subsidiario, ya que procede su utilización exclusivamente en las hipótesis más complejas, donde no emerja una competencia activa exclusiva de un único Ente público. Esta regla se fundamenta, básicamente, en el estudio de dos tipos de datos: el examen de la financiación de los acuerdos de programa, y el análisis de su ámbito espacial. El primer examen consiste en realizar una valoración económico-cuantitativa de la específica participación de las diferentes Administraciones en un determinado acuerdo de programa, mientras que el segundo consiste en determinar cuál es el ámbito territorial donde dicho acuerdo tendrá mayor incidencia $^{22}$. El problema aplicativo más espinoso que plantea este criterio de la prevalencia radica en determinar cómo conjugar los resultados de la realización de ambos estudios, ya que dada la extrema variedad de hipótesis que se pueden verificar, no será infrecuente, que del análisis de los resultados de ambos exámenes se puedan inferir soluciones diversas ${ }^{23}$. Así las cosas, dado que no es posible dar una respuesta de validez general, es ineluctable afirmar que la prevalencia debe ser determinada, caso por caso, utilizando de forma sincrética los criterios que parezcan razonablemente aplicables a cada concreto supuesto ${ }^{24}$.

${ }^{21}$ Cfr. G. Greco, «Commento all'art. 27», op. cit., p. 390; G. SAntomauro, «L'accordo di programma: procedimento di formazione ed approvazione - natura giuridica- effetti ed esecuzione», Com. it., n. ${ }^{\circ}$ 9, 1992, p. 1329; G.D. COMPORTI, Il coordinamento infrastrutturale, op. cit., p. 282. Ciertas Leyes regionales han individuado determinados supuestos en los que concurre un interés regional prevalente, así, p. ej., la L. r. Puglia n. ${ }^{\circ}$ 4/1995, de 20 de febrero, Disciplina delle procedure per gli accordi di programma, en su art. 1, apartado 2, dice: «Ai fini di cui al precedente comma 1 sono ritenuti di prevalente interesse regionale in particolare, le opere, gli interventi ed i programmi previsti dal piano regionale di sviluppo, gli altri piani e programmi regionali di settore, nonché quando ne ricorrono le condizioni, le iniziative finanziate con leggi e programmi nazionali e comunitari» (en idénticos términos se pronuncia la L. r. Molise n. ${ }^{\circ} 17 / 1999$, de 11 de junio, Disciplina del procedimento per l'accordo di programma).

22 Cfr. F. CAIFFA, «Le nuove frontiere dell'attività amministrativa consensuale: gli accordi di programa», op. cit., p. 234; G. D. COMPORTI, Il coordinamento infrastrutturale, op. cit., p. 282; A. CIMELLARO y A. FERRUTI, La conferenza di servizi e l'accordo di programma, $2^{\mathrm{a}}$ ed., Maggioli, Rimini, 2000, p. 142. Vid., también, S. TAR Marche, de 5 de noviembre de 1999, n. 1264.

${ }^{23}$ De este modo, mientras que según los resultados del primer análisis el sujeto promotor será aquel económicamente más interesado en la ejecución del acuerdo de programa, es decir, el que aporte una mayor financiación al mismo; de acuerdo con los datos obtenidos en el segundo, el sujeto promotor será aquel en cuyo territorio se haya de realizar la mayor parte de las actividades previstas en dicho acuerdo.

24 Vid. E. Sticchi DAmí́n, Attività amministrativa consensuale e accordi di programma, op. cit., p. 184. En definitiva, y como señala Beniamino Caravita, se trata de parámetros de no cómoda aplicación. Reglas que no permiten excluir que la incertidumbre sobre la determinación del sujeto pro- 
Para terminar con el análisis del procedimiento de individuación del sujeto titular del poder de promoción, hay que señalar que en el apartado 8 del art. 34 TUEL se establece una excepción al procedimiento general que se acaba de exponer ${ }^{25}$. A través de este precepto, en la hipótesis en que un acuerdo de programa implica a varias Regiones limítrofes, el Legislador atribuye el poder de promoción directamente, sin necesidad de que los operadores jurídicos lleven a cabo ninguna actividad de concreción, a la Presidencia del Consejo de Ministros ${ }^{26}$.

\section{LOS ASPECTOS OBJETIVOS DE LOS ACUERDOS DE PROGRAMA}

El estudio de la vertiente objetiva de los acuerdos de programa comprende, por una parte, el análisis de su objeto, con el fin de determinar cuál es la realidad social situada en el ámbito de ejercicio de las competencias propias de los entes suscriptores, sobre la cual dichos acuerdos, en cuanto negocios jurídicos, pueden incidir, y, por otra parte, el examen de su contenido, es decir, de las prescripciones que deben o pueden incluirse en los acuerdos de programa para disciplinar las condiciones en las que éstos van a actuar sobre la realidad social que constituye su objeto.

\section{1) El objeto de los acuerdos de programa}

El art. 34 TUEL enuncia, de forma no muy clara, el objeto de los acuerdos de programa. Concretamente, dice que éstos se pueden emplear para la definición y la actuación de obras, de intervenciones o de programas de

\footnotetext{
motor pueda paralizar el proceso de elaboración de los acuerdos de programa («Gli accordi di programa», op. cit., p. 39).

${ }^{25}$ Literalmente este precepto dice: «Allorché l'intervento o il programma di intervento comporti il concorso di due o più regioni finitime, la conclusione del accordo di progranuna è promossa dalla Presidenza del Cosiglio dei Mistri, a cui spetta convocare la conferenza di cui al comma $3 »$.

${ }^{26}$ Gian Domenico COMPORTI entiende que el Legislador se refiere exclusivamente a los acuerdos concluidos sólo entre Regiones colindantes. En su opinión, en el caso en que sean parte del acuerdo otros Entes públicos o Regiones no colindantes, debe aplicarse el criterio general (Il coordinamento infrastrutturale, op. cit., p. 266).
}

La doctrina estima que con esta previsión el Legislador ha querido reservar un papel arbitral a la Presidencia del Consejo de Ministros, con el fin de permitirle dirimir eventuales conflictos que pudieran perjudicar el interés nacional (cfr. R. FERRARA, Gli accordi di programma, op. cit., p. 105; G.D. COMPORTI, Il coordinamento infrastrutturale, op. cit., pp. 266-267). 
intervención ${ }^{27}$. Se trata de una descripción voluntariamente amplia y genérica, que revela la clara intención del Legislador italiano de no limitar el recurso a este instrumento de coordinación y simplificación de la acción administrativa ${ }^{28}$. Dada esta laxa formulación legal, parece que el mejor modo de abordar el estudio del objeto de los acuerdos de programa es proceder al análisis detallado de cada uno de los términos que emplea el TUEL, tratando de precisar cuál es su contenido en este contexto concreto. Así, en primer lugar, se debe comenzar por explicar el significado y la relación existente entre los términos: definición y actuación. Definir consiste en fijar con claridad, exactitud y precisión una determinada materia. Por ello, prima facie, se puede pensar que hace referencia a la fase de planificación de una determinada, actividad administrativa. A su vez, actuar significa poner en acción una determinada cosa. Por lo que, esta expresión parece referirse a la fase ejecutiva de dicha actividad. Entre ambos términos no es adecuado establecer una estricta relación de coordinación, ya que no es práctico sostener que los acuerdos de programa deban ser, necesariamente, en todo momento, actos de programación y de ejecución de las actividades públicas. Por el contrario, parece más oportuno entender que entre dichos términos existe, en alternancia con el coordinativo, un nexo disyuntivo. De este modo, los acuerdos de programa puede tener por objeto, bien la programación de actividades, bien la ejecución de intervenciones ya programadas, o bien una y otra posibilidad combinadas $^{29}$. Una vez que se ha precisado el significado y la relación existente entre los términos definición y actuación, hay que pasar a examinar el alcance de las tres siguientes expresiones: obras, intervenciones y programas de intervenciones. Así, en primer lugar, parece que el Legislador italiano con el término obras hace referencia al concepto de obra pública, de modo que queda comprendida dentro del objeto de los acuer-

\footnotetext{
${ }^{27}$ El tenor literal del art. 34.1 TUEL dice: «[...] per la definizione e l'attuazione di opere, di interventi o di programmi di intervento $[\ldots]$ ».

${ }^{28}$ Así lo ha entendido el Consiglio di Stato, el cual en su S. Cons. Stato, Sez. IV, de 1 de agosto de 2001, n. ${ }^{\circ} 4206$, afirma: «L'individuazione dell'ambito oggettivo in modo ampio, ed anche generico, evidenzia l'intenzione del legislatore di rendere il più possibile generale lo strumento dell'accordo di programma».

29 Vid. M. Pallottino, «Strumenti giuridici di concertazione amministrativa: L'accordo di programma», Riv. amm, fasc. 12, 1983, p. 848; G. MANFREDI, «Modelli contrattuali dell'azione amministrativa: l'accordo di programma», op. cit., p. 362; F. MANGANARO, «Rilievi sugli accordi tra pubbliche amministrazioni», Scritti per Enzo Silvestri, Giuffrè, Milano, 1992, pp. 375-376; R. FERRARA, Gli accordi di programa, op. cit., p. 90; G.D. COMPORTI, Il coordinamento infrastrutturale, op. cit., p. 275. En contra de esta postura se ha pronunciado Guido GRECO quien sostiene que «[...] il c.d. accordo di programma di per sé non ha nulla di programmatorio, avendo viceversa compiti e finalità tipicamente deliberativi ed attuativi [...]» («Commento all'art. 27», op. cit., p. 386).
} 
dos de programa cualquier tipo de modificación del mundo físico $\mathrm{y}$, en especial, cualquier tipo de construcción que interese a los poderes públi$\cos ^{30}$. En segundo lugar, puede interpretarse el vocablo intervenciones en el sentido de que el mismo se refiere a otras actividades administrativas diferentes de las obras públicas, p. ej., financieras ${ }^{31}$. Con esta interpretación que equipara, en la práctica, intervenciones a casi cualquier tipo de actuación administrativa, se puede sostener, como hace Aldo TRAVI, que los acuerdos de programa pueden tener como objeto tanto la gestión de servicios como el desempeño de funciones administrativas ${ }^{32}$. En tercer

30 «Cfr. M.S. Giannini, Diritto pubblico dell'economia, Il Mulino, Bologna, 1993, p. 51. En este punto, Guido GRECO precisa: «[...] a me non pare che le opere statali siano escluse dall'acoordo di programma di cui al art. 27. Se così non fosse, non si comprenderebbe il riferimento, contenuto nel comma 4 del medesimo articolo, all'art. 81 del DPR n. ${ }^{\circ} 616$ del 1977, che riguarda, com'è noto, l'intesa che intercorre tra Stato e Regioni per opere di interesse statale» («Accordi di programma e procedimento amministrativo», I rapporti fra cittadini e istituzioni nelle recenti leggi di riforma delle autonomie locali e del procedimento amministrativo. Atti del Convegno di Milano, 14 gennaio 1991, Giuffrè, Milano, 1992, p. 51). En este sentido hay que tener en cuenta, además, que la jurisprudencia ha señalado que puede ser objeto de un acuerdo de programa, sólo la realización de obras públicas, sino también la ejecución de obras privadas, a condición de que hayan sido juzgadas de interés público (vid. S. TAR Lazio, Sez. I, de 20 de enero de 1995, n. ${ }^{\circ}$ 65; S. Cons. Stato, Sez. VI, de 7 de febrero de 1996, n. ${ }^{\circ} 182$ ), esta orientación jurisprudencial. ha sido recogida por algunas Leyes regionales, como, p. ej., la L. r. Valle d'Aosta n. ${ }^{\circ}$ 11/1998, de 6 de abril, Normativa urbanistica e di pianificazione territoriale della Valle d'Aosta, algunas de ellas, como p. ej., la L. r. Liguria n. 36(1997, de 4 de septiembre, Legge urbanistica regionale, establecen ciertos límites y condiciones.

31 Así, según Guido GRECO: «[...] si può dire che entrano nella fattispecie del art. 27 (della L. n. 142) i casi di partecipazione integrata di più autorità, diretta al compimento di prestazioni amministrative (appunto, opere o altro tipo di interventi). [...] Data l'ampia porta normativa dell'art. 27, inoltre, si può ritener che esso possa applicarsi ad ogni tipo di attività diretta alla realizzazione di opere e ai connessi interventi [...] Non è escluso, inoltre, che l'art 27 possa trovare applicazione anche per la programmazione e realizzazlone di interventi (ad esempio finanziari), non direttamente connessi con il compimento di opere» («Accordi di programma e procedimento amministrativo», op. cit., pp. 49-50).

32 Vid., en este sentido: A. Travi, «Le forme di cooperazione interlocale», Dir. Amm., n. ${ }^{\circ}$ 4, 1996, p. 688; S. Civitarese Matteucci, «Accordo, di programma», op. cit., p. 10, n. ${ }^{\circ}$ 2. En la S. Cons. Stato, Sez. IV, de 1 de agosto de 2001, n. ${ }^{\circ} 4206$, el Consiglio di Stato realiza una interpretación distinta: "Infatti, il termine "interventi o programmi di intervento", inserito nell'art. 27, comma 1 , in aggiunta alla «definizione e attuazione di opere» deve essere interpretato come possibilità di utilizzo dello strumento non solo per qualsiasi tipo di opera puliblica, ma anche per la programmazione di attività ulteriori e complementari rispetto alla realizzazione delle opere (come, nel caso di specie, i previsti interventi a sostegno dell'occupazione)». Otra posibilidad interpretativa de estos términos es la realizada por la L. r. Valle d'Aosta n. ${ }^{\circ}$ 11/1998, de 6 de abril, Normativa urbanistica e di pianificazione territoriale della Valle d'Aosta, en su art. 26.4. Según este precepto: «Ai fini della determinazione del possibile contenuto degli acoordi di programma, per interventi si intendono gli insiemi sistematici di opere o di azione concrete; per programmi di intervento si intendono gli atti che, considerando un insieme sistematico di interventi, coordinano gli stessi anche nel tempo, prevedono le fonfi di finanziamento e i mezzi di attuazione ed in genere predispongono quanto occorre operativamente per la realizzazione degli interventi medesimi». 
lugar, con la expresión programas de intervenciones el Legislador italiano, según la doctrina, se refiere con toda probabilidad, no a una singular actividad pública, para lo que hubiese podido emplear cualquiera de las expresiones precedentes, sino a una pluralidad de actuaciones públicas que integran un auténtico proyecto, es decir, un acto que, sobre la base de los recursos iniciales y de un detallado inventario de las disponibilidades presentes y futuras, fija para una fecha final objetivos concretamente realizables y establece las concretas líneas de conducta que permiten alcanzar dichos objetivos ${ }^{33}$.

En resumen, a la luz de lo expuesto, se puede concluir que el objeto de los acuerdos de programa puede consistir, bien en la programación y/o ejecución de concretas obras públicas o de otro tipo de actuaciones administrativas, o bien en la programación y/o ejecución de un conjunto de ellas.

\section{2) El contenido de los acuerdos de programa}

También a la hora de disciplinar el contenido de los acuerdos de programa, el Legislador italiano ha querido dejar a las partes una amplia facultad de determinación del mismo, en función de su valoración de lo que es más adecuado a las exigencias de cada caso. Dentro de dicha regulación del contenido de los acuerdos de programa es posible distinguir tres grandes subconjuntos: el contenido necesario, que es aquel directamente establecido por el Legislador y que las partes siempre deben incluir en los acuerdos de programa; el contenido facultativo que está integrado por un conjunto de cuestiones que el Legislador relaciona, pero cuya inserción o no en cada concreto acuerdo de programa deja a la libre voluntad de las partes; y, finalmente, el contenido eventual, el cual está constituido por todas aquellas disposiciones que los participantes introducen en cada singular acuerdo, con el fin de lograr que éste sea el mejor instrumento posible para lograr los objetivos que con él se proponen alcanzar.

De acuerdo con el apartado primero del art. 34 TUEL, integran el contenido necesario de los acuerdos de programa: en primer lugar, la especificación del objeto, es decir, el detalle de las obras o intervenciones que se quieren realizar mediante la suscripción de dicho acuerdo; en segundo lugar, la enumeración de los fines que se pretenden alcanzar con el mismo; en tercer lugar, la determinación de los plazos, que consiste tanto

\footnotetext{
33 El concreto significado de este vocablo se debe buscar en el lenguaje propio de los economistas; por ello, vid.: P.A. SAmuelson y W. D. Nordhaus, Economía, 14 a ed., MacGraw-Hill, Madrid, 1994.
} 
en señalar el plazo de vigencia del acuerdo de programa, como en establecer el proyecto de ejecución del objeto del acuerdo, en donde las actuaciones que se llevarán a cabo aparecen divididas en fases, con indicación de los tiempos respectivos; en cuarto lugar, la explicación de la financiación, que consiste, por un lado, en fijar cuál será coste global de las actuaciones, especificando qué parte corresponde a cada una de las fases de ejecución, y, por otro lado, en determinar cuáles serán las fuentes de financiación para hacer frente a ese coste ${ }^{34}$; en quinto lugar, la determinación de la forma de ejecución del acuerdo de programa, es decir, el modo en que se piensa acometer la realización de las intervenciones previstas en el mismo; y, en sexto, y último lugar, la fijación de la disciplina del colegio de vigilancia, a quien corresponde la supervisión de la ejecución del acuerdo de programa; esencialmente, se debe determinar su composición y detallar las normas que van a regir su funcionamiento ${ }^{35}$.

Además, el legislador ha dejado al arbitrio de las partes en cada acuerdo de programa la posibilidad de establecer procedimientos de arbitraje y de sustitución en caso de incumplimiento. Ambas posibilidades constituyen el llamado contenido facultativo de los acuerdos de programa $^{36}$.

${ }^{34}$ Con la inclusión de estos datos económicos, los acuerdos de programa se convierten en auténticos planes financieros de las actuaciones a realizar. Esta decisión del Legislador italiano, como resalta Francesco MANGARANO, se revela muy oportuna, ya que el retraso en la programación y ejecución de muchas intervenciones públicas se debe, generalmente, a la falta de previsión de las fuentes de financiación y de un claro reparto de las cargas financieras entre los Entes participantes («Rilievi sugli acoordi tra pubbliche amministrazioni», op. cit., p. 377).

35 Algunas Leyes regionales configuran un contenido obligatorio más amplio, así, p. ej., la L. r. Toscana n. ${ }^{\circ} 76 / 1996$, de 3 de septiembre, Disciplina degli accordi di programma e delle conferenze dei servizi, exige, en su art. 4.1, además de lo expuesto: «[...] c) l'indicazione del funzionario responsabile dell'attuazione dell'accordo; le modalità del controllo ai fini dell'attuazione dell'accordo, i provvedimenti sanzionatori da adottarsi in caso di inadempimento, inerzia o ritardo; d) l'eventuale ricorso a procedimenti di arbitrato con la specificazione delle modalità di composizione e di nomina del collegio arbitrale; e) le eventuali variazioni di atti di programmzione o pianificazione territoriale determinate dall'accordo di programma, nel rispetto delle procedure previste dall'art. 11; f) l'eventuale effetto sostitutivo delle concessioni edilizie, di cui all'art. 27, comma 4,1. 8/6/1990 n. ${ }^{\circ} 142$ ed ogni eventuale altro effetto sostitutivo di atti amministrativi, secondo le disposizioni della presente legge». Junto con estas previsiones, la L. r. Lombardia n. 2/2003, de 14 de marzo, Programmazione negoziata regionale, en su art. 6.7, requiere que en el acuerdo de programa se expresen: «[...] e) gli adempimenti attribuiti ai soggetti interessati, le responsabilità dell'attuazione e le eventuali garanzie; f) le sanzioni per gli inadempimenti [...]».

${ }^{36}$ Literalmente el Legislador italiano habla de «[...] procedimenti di arbitrato e di interventi surrogatori in caso di inadempienza [...]». La previsión de procedimientos arbitrales se ha mostrado, sin duda, útil para resolver en sede extrajudicial, de forma rápida, los posibles conflictos que puedan surgir en relación con la interpretación y ejecución de los acuerdos de programa. A su vez, las intervenciones sustitutivas representan un remedio de excepcional eficacia para resolver situaciones patológicas de bloqueo que puedan surgir en la ejecución de los acuerdos de programa. 
Por último, el art. 34.1 in fine oportunamente remite a la voluntad de las partes el incorporar al contenido de los acuerdos de programa cualquier otra obligación que puedan considerar necesaria para la completa realización del objeto de los mismos ${ }^{37}$. El conjunto de estas posibles previsiones constituye el contenido eventual de los acuerdos de programa ${ }^{38}$.

\section{LOS ASPECTOS PROCEDIMENTALES DE LOS ACUERDOS DE PROGRAMA}

En la vida de todo acuerdo de programa hay que distinguir, por una parte, su proceso de formación, que engloba todos los actos que preceden a su suscripción y exteriorización, momentos que marcan el inicio de la producción de sus efectos, $y$, por otra parte, su fase de ejecución, que consiste tanto en la actuación de todas las actividades previstas en el mismo, como en la fiscalización de dicha actuación.

\section{1) La formación de los acuerdos de programa}

A su vez, dentro del proceso de configuración de los acuerdos de programa se pueden individuar tres fases distintas: la fase de instrucción, la fase de decisión y la fase de aprobación.

\section{A) La fase de instrucción}

El TUEL prevé que esta fase se lleve a cabo mediante la celebración de una conferencia, es decir, de una reunión de los representantes de todos los entes interesados ${ }^{39}$. El objetivo de esta conferencia es verificar la posi-

\footnotetext{
${ }^{37}$ El tenor literal de este precepto dice: «[...] ed ogni altro connesso adempimento [...].

${ }^{38}$ De entre las múltiples estipulaciones que pueden constituir el contenido eventual de los acuerdos de programa, merece la pena destacar, por su utilidad, dos: en primer lugar, es posible que las Administraciones participantes pacten, o bien constituir organismos de consulta técnica, con la función de coadyuvarles en la gestión del acuerdo, o bien atribuir tales competencias a sociedades de servicios o ingeniería (cfr. F. MANGANARO, «Rilievi sugli accordi tra pubbliche amministrazioni», op. cit., p. 378); en segundo lugar, las Administraciones partes en un acuerdo de programa pueden establecer en el mismo las bases para la suscripción de futuros convenios (como, por ejemplo, los pactos con particulares de los que se ha hablado en el apartado II.1 del presente trabajo).

39 Esta conferencia es un instituto distinto de aquél de alcance general, conocido como conferenza di servizi, regulado en el art. 14 de la L. n. ${ }^{\circ}$ 241/1990, 7 agosto, Nuove norme in materia di procedimento amministradvo e di diritto di accesso ai documenti amministrativi. La diferencia más nota-
} 
bilidad de suscribir un sucesivo acuerdo de programa. En consecuencia, en ella las Administraciones convocadas tratan de determinar si concurren las condiciones necesarias para celebrar un subsiguiente acuerdo de programa, para lo que proceden a valorar el grado de eficacia de tal posible acuerdo como instrumento para la satisfacción de determinados intereses cuya consecución tienen encomendada. Ello les exige, necesariamente, ponderar los costes y los beneficios, tanto en términos económicos como sociales, que implica dicho acuerdo para cada una de ellas.

Para una adecuada compresión de cómo se desarrolla la formación de los acuerdos de programa, conviene analizar tanto la convocatoria, como el funcionamiento de esta conferencia.

\section{a) La convocatoria de la conferencia}

El TUEL no disciplina el modo en que ha de llevarse a cabo la convocatoria de la conferencia ${ }^{40}$. Ante este silencio, la solución que parece más adecuada es que la Administración a quien corresponde el poder de promoción convoque a las demás Administraciones previsiblemente interesadas mediante el envío de una propuesta de acuerdo de programa. En dicha propuesta, en primer lugar, habrá de contenerse un proyecto o esquema de acuerdo. Éste tendrá que estar integrado, al menos, por un esbozo de la actuación administrativa que va a constituir el objeto del acuerdo de programa, y por la especificación de las obligaciones que, para su realización, deberá afrontar cada una de las posibles Administraciones participantes ${ }^{41}$. En segundo lugar, en la citada propuesta habrá de señalarse la fecha y el lugar en la que se celebrará la conferencia. A la hora de determinar el momento en el que tendrá lugar dicha conferencia, es necesario que la Administración convocante tenga en cuenta que se debe conceder a las

ble entre ambos radica en que en esta conferencia es necesaria la unanimidad de los participantes para adoptar una decisión, mientras que en la conferencia regulada por el art. 14 de la citada L. n. ${ }^{\circ} 241 / 1990$ se prevé, en general, la regla de la mayoría, salvo las excepciones previstas para cuestiones de particular interés, como aquellas de carácter ambiental, histórico-artístico y sanitario. Sobre las conferencias de servicios, vid., entre otros: P.M. PIACENTINI, «La conferenza di servizi: considerazioni generali», Riv. trim. appalti, n. ${ }^{\circ}$ 4, 1989, pp. 1131 ss.; M.A. QUAGLIA, «La conferenza di servizi: profili procedimentali», Quad reg., n. ${ }^{\circ} 1-2,1991$, pp. 105 ss.; P. BERTINI, «La conferenza di servizi», Dir amm., n. ${ }^{\circ} 2,1997$, pp. 325 ss.

${ }^{40}$ El art. 34.3 TUEL tan sólo dispone: «Per verificare la possibilità di coneordare l'accordo di programma, il presidente della regione o il presidente della provincia o il sindaco convoca una conferenza tra i rappresentanti di tutte le ammministrazioni interessate».

${ }^{41}$ Cfr. Presidenza del consiglio dei ministri-Departamento della funcione pubblica, «Gli accordi di programa», op. cit., p. 61. 
Administraciones invitadas un plazo razonable para el estudio y valoración del material que se les ha remitido ${ }^{42}$.

\section{b) El funcionamiento de la conferencia}

El TUEL no especifica tampoco cuál será el régimen de funcionamiento de la conferencia inicial, es decir, no establece las normas conforme a las cuáles debe desarrollarse la negociación entre las Administraciones participantes en dicha conferencia ${ }^{43}$. Es posible entender que esta ausencia de regulación determina el reconocimiento de una amplia libertad a las mismas. No obstante, si bien es cierto que se concede a las Administraciones un ámbito de libertad extenso, también lo es que éste no es ilimitado. Así, es patente que, con el establecimiento de la obligación de celebrar una conferencia, el Legislador pretende privilegiar el examen contextual, directo y oral, de la posibilidad de celebrar un acuerdo de programa. En consecuencia, parece que queda excluida la posibilidad de que se lleve a cabo un estudio aislado de la propuesta de acuerdo por parte de cada uno de los sujetos individuados por el sujeto promotor. Por esta misma razón, tampoco será posible que los sujetos públicos que intervienen en la conferencia negocien en mesas separadas ${ }^{44}$.

\footnotetext{
${ }^{42}$ Frente a la falta de concreción en este punto de la Ley estatal, en algunas Leyes regionales se especifican detalladamente los contenidos mínimos de la propuesta de acuerdo. Así, el apartado 2 del art. 2 de la L. r. Lombardia n. ${ }^{\circ}$ 2/2003, de 14 de marzo, Programmazione negoziata regionale, establece: «La proposta: a) indica le opere i programmui, gli interventi, gli obiettivi generali degli stessi, e l'ámbito territoriale, b) individua i soggetti di cui al comma 1 dei quali sia prevista l'azione integrata; e) fissa il termine entro il quale deve essere definito l'accordo di programa». Una regulación similar se puede encontrar también en los artículos: 8 bis apartado 4 de la L r. Abruzzo 18/1983, de 12 de abril, Norme per la conservazione, tutela trasformazione del territorio della Regione Abruzzo, en la redacción que le ha dado el art. 6 de la L. r. Abruzzo 70/1995, de 27 abril, Modifiche ed integrazioni alla L. R. 12-4-1983, n. ${ }^{\circ}$ 18: Norme per la conservacione, tutela, trasformazione del territorio della Regione Abruzo; 2 apartado 2 de la L. r. Puglia n. ${ }^{\circ}$ 4/1995, de 20 de febrero, Disciplina delle procedure per gli accordi di programma; y 7 apartado 4 de la L. r. Toscana n. ${ }^{\circ} 76 / 1996$, de 3 de septiembre, Disciplina degli accordi di programma e delle conferenze di servizi.

43 Según la jurisprudencia, las Administraciones invitadas a participar en la conferencia tienen el deber jurídico de participar en ella. En consecuencia, no pueden legítimamente rehusar participar en la misma sobre la base de una apriorística toma de posición negativa, aunque, naturalmente, podrán expresar el propio disenso con la propuesta de acción concordada (vid., S. TAR Lombardia, Sez. II de 30 de marzo de 1996. n. ${ }^{\circ}$ 417). Cfr. N. BASSI, «Commento all'articolo 34», op. cit., p. 407.

${ }^{44}$ Cfr. S. Civitarese Matteucci, «Accordo di programa», op. cit., p. 16. Giuseppe Manfredi se muestra contrario a este sistema de negociación, ya que en su opinión: «L'esperienza di chiunque abbia partecipato ad una qualsivoglia forma di contrattazione multilaterale potrebbe confermare che, con questa nuova "rigidità", le chances di addivenire ad un accordo diminuiscono sensiblemente. Anche in questo caso la ratio pare essere quell'esaperato garantismo verso i diversi interessi pubblici che anima il legislatore quando ha a che fare con gli acoordi di programma» («Modelli contrattuali dell'azione amministrativa: l'accordo di programa», op. cit., p. 369).
} 
Por el contrario, nada impide que la conferencia articule su trabajo en varias sesiones ${ }^{45}$.

Es necesario destacar, respecto al comportamiento de las Administraciones que participan en esta conferencia, que éstas están obligadas únicamente a negociar, y no a pactar. Esta obligación de negociar implica que dichas Administraciones no pueden realizar un mero examen conjunto, o una simple consulta, sino que tienen que desarrollar una verdadera actividad de negociación que se debe desarrollar de acuerdo con los principios de corrección y buena fe.

El resultado último que el TUEL persigue al introducir esta conferencia, en la que se realiza una preventiva verificación de la posibilidad de concluir un acuerdo de programa, es que, al final de ésta, las Administraciones que intervienen en la misma asuman una especie de predecisión que las vincule a franquear el camino del acuerdo. Se trata de una predeterminación que influye sobre el an, pero que no condiciona el quomodo del acuerdo mismo. Dicho de otro modo, con ella las Administraciones participantes se limitan a fijar su intención de concluir un futuro acuerdo de programa, del cual pueden indicar las características básicas, dejando, en principio, imprejuzgado cualquier otro pronunciamiento sobre el contenido del mismo ${ }^{46}$. No es infrecuente que esta predecisión se materialice en una intesa di programma. Ésta constituye un acto que acredita la decisión política de los Entes públicos implicados de alcanzar un acuerdo. Se trata de una especie de acuerdo preliminar sobre los objetivos y finalidades estratégicos de fondo, que carece de capacidad para vincular la concreta definición de las cláusulas del acuerdo definitivo ${ }^{47}$.

\footnotetext{
${ }^{45}$ Hay que señalar que es habitual que de los trabajos de esta conferencia se levante acta. Así, el art. 8 de la L. r. Toscana n. ${ }^{\circ}$ 76/1996, de 3 de septiembre, Disciplina degli accordi di programma e delle conferenze di servizi, dispone: «3. Della conferenza viene redatto apposito verbale, a cura del responsabile del procedimento, dal quale devono risultare i soggetti presenti, le determinazioni assunte in ordine alla prosecuzione del procedimento per la conclusione dell'accordo di programma ed i tempi e le modalità di espletamento degli eventuali adempimenti istruttori necessari. Il verbale deve essere sottoscritto da tutti i partecipanti. 4. Il verbale della conferenza è allegato all'accordo di programma».

46 Vid. R. FerRaRA, Gli accordi di programma, op. cit., p. 113; G.D. ComporTI, Il coordinamento infrastrutturale, op. cit., pp. 283-284.

${ }^{47}$ Carlo Modica sostiene que de esta intesa de programma pueden surgir vínculos jurídicos que superan el mero compromiso político. Según este autor, ésta puede implicar, por ejemplo, el compromiso de asignar los recursos económicos necesarios para realizar los estudios de factibilidad de la actuación pública objeto del futuro acuerdo de programa («L'accordo di programma: istituzionalizzazione dell'inefficienza?», Riv. giur mezz., n. ${ }^{0} 1,1991$, p. 26).
} 
Sin embargo, no es posible excluir, dado que lo que prevalece en todo este procedimiento negocial es la voluntad de las partes, que, en esta fase preliminar, se pueda llegar a un concierto más amplio, y se consiga, incluso, el consenso sobre el acuerdo de programa final ${ }^{48}$.

\section{B) La fase de decisión}

Si en la conferencia inicial que se acaba de estudiar sólo se llegó al consenso sobre la conveniencia de celebrar un posterior acuerdo de programa, es necesario que, tras ella, se abra una nueva fase cuyo objetivo será la definición y plasmación del contenido concreto de dicho acuerdo.

En esta nueva etapa del proceso de gestación de los acuerdos de programa se plantean varios interrogantes: en primer lugar, cuál es el procedimiento que han de seguir las Administraciones para llegar a su formulación definitiva; y, en segundo lugar, cuáles de ellas deben adherirse y suscribir dichos acuerdos para que éstos sean válidos.

\section{a) La determinación del contenido de los acuerdos de programa}

Para realizar esta concreción parece lógico que se convoque, en la forma antes estudiada, una nueva conferencia ${ }^{49}$. En el seno de esta nueva conferencia ha de desarrollarse una intensa actividad de negociación con el fin de llegar, mediante la confrontación de las propuestas y contrapropuestas que formulen las diferentes Administraciones interesadas en el

\footnotetext{
${ }^{48}$ Cfr. G.D. COMPORTI, Il coordinamento infrastrutturale, op. cit., p. 284. Tampoco cabe descartar la posibilidad de que se produzca un acuerdo parcial, es decir, que se alcance el consenso sobre algunas cuestiones, pero no sobre otras. En este caso, aunque no puede considerarse formado el acuerdo de programa, este pacto inacabado constituye un vínculo entre las partes, desde la perspectiva del respeto a la buena fe, $\mathrm{y}$, en consecuencia, la pretensión de cualquiera de ellas de desatenderlo puede implicar la responsabilidad precontractual de quien así actúe (cfr. G. PERULLI, «L'iter preliminare all'accordo di programa», Riv. trim. appalti, n. ${ }^{\circ}$ 4.1992, pp. 736-737).

49 A este respecto, Guido GRECO afirma: «Altra soluzione potrebbe essere quella di indire più di una conferenza; la prima, ad esempio, di carattere informativo e in senso lato istruttorio, la seconda di carattere decisionale (e, se risulti necessario apportare modifiche al progetto di accordo, non è da escludersi ulteriori convocazioni). Né si pensi che, così procedendo, risulti frustrata del tutto la funzione acceleratoria dell'istituto: ques'ultima, infatti, risulta pur sempre salvaguardata dalla cadenza delle convocazioni della conferenza e dalla semplificazione che deriva da un contatto diretto delle varie amministrazioni interessate» («Commento all'art. 27», op. cit., p. 394). En este mismo sentido, se ha manifestado Giovanni SANTOMAURo («L'accordo di programma: procedimento di formazione ed approvazione -natura giuridica- effetti ed esecuzione», op. cit., p. 1330).
} 
acuerdo, a una redacción consensuada del texto definitivo del acuerdo de programa $^{50}$.

Algunas Leyes regionales establecen con idéntica finalidad que, una vez que en la conferencia inicial, las Administraciones participantes en la misma han llegado a la convicción de que es posible concluir un acuerdo de programa, se debe constituir un comité encargado de su redacción ("comitato per l'accordo di programa") ${ }^{51}$. Normalmente, forman parte de dicho comité los representantes de las Administraciones y de los Entes interesados en alcanzar el acuerdo de programa, o sus delegados, correspondiendo la presidencia del mismo al representante de la Administración promotora $^{52}$. Su función consiste en consensuar, teniendo en cuenta los intereses de las Administraciones participantes, las actuaciones que deben constituir el objeto del acuerdo de programa, determinando específicamente el coste y las fuentes de financiación de las mismas. Para llegar a tal resultado dicho comité elabora y coordina distintas propuestas técnicas y financieras, mediante la realización de los oportunos estudios y verificaciones, pudiendo solicitar, si lo estima preciso, consultas técnicas especializadas. En la realización de esta labor, el citado comité puede contar con el apoyo de una secretaría técnica compuesta por funcionarios procedentes les de las Administraciones participantes ${ }^{53}$.

\section{b) La adhesión al acuerdo de programa y la suscripción del mismo}

Una vez que se ha consensuado una redacción del acuerdo de programa que contenta a los sujetos que han intervenido en la negociación, ésta

\footnotetext{
${ }^{50}$ No obstante, no hay que olvidar que, a lo largo de este proceso, puede suceder que una de las Administraciones participantes no se muestre conforme con la evolución de las negociaciones, por entender que el acuerdo hacia el que se camina no satisface los intereses cuya tutela tiene encomendada. En este caso, dicha Administración deberá manifestar, formal y motivadamente, el propio disenso y, si lo considera oportuno, podrá abandonar el procedimiento.

${ }^{51}$ Cfr. L. r. Pluglia n. ${ }^{\circ} 4 / 1995$, de 20 de febrero, Disciplina delle procedure per gli accordi di programma; la L. r. Molise n. ${ }^{\circ}$ 17/1999, de 11 de junio, Disciplina del procedimento per l'accordo di programma; la L. r. Lombardia n. ${ }^{\circ} 2 / 2003$, de 14 de marzo, Programmazione negoziata regionale.

${ }^{52}$ Estas norams suelen reconocer la posibilidad de que a este comité puedan adherirse, en un momento posterior, otros sujetos públicos interesados en suscribir el acuerdo de programa. Sin embargo, comúnmente, se establece una limitación: la adhesión de estos entes no puede conllevar una interrupción del proceso de decisión que se halla en marcha.

${ }^{53}$ En algunas leyes regionales, en lugar de preverse la institución de un comité, se dispone el nombramiento de un responsable del procedimiento. Éste debe proceder a formular el texto definitivo del acuerdo de programa de tal forma que dicho texto sea aceptado por todas las Administraciones implicadas (cfr. art. 27.1 de la L. r. Valle d'Aosta n. ${ }^{\circ}$ 11/1998, de 6 de abril, Normativa urbanistica e di pianificazione territoriale della Valle d'Aosta).
} 
finaliza y llega el momento de la conclusión de dicho acuerdo. Momento que consiste en la manifestación de la adhesión al mismo por parte de todas las Administraciones interesadas ${ }^{54}$.

Hay que señalar que los acuerdos de programa sólo pueden ser estipulados, como señala el art. 34 TUEL, con el consenso unánime de las Administraciones interesadas. Así las cosas, la doctrina ha discutido sobre qué debe entenderse por el consenso unánime de las Administraciones interesadas, al que hace referencia el TUEL. Para un sector, esta expresión exige la adhesión incondicionada a los acuerdos de programa de todos los sujetos invitados a la conferencia inicial ${ }^{55}$. Si se sigue esta interpretación este instituto deviene poco funcional, ya que todos los sujetos convocados dispondrían de un injustificado poder de veto sobre dichos acuerdos ${ }^{56}$. Por el contrario, otro grupo de autores, con mejor criterio, entiende que no es

\footnotetext{
${ }^{54}$ En este punto, la doctrina se ha planteado si la conclusión de un acuerdo de programa puede determinar una alteración del reparto ordinario de competencias entre los órganos de los Entes locales, habida cuenta del principio de celeridad de la actuación administrativa que inspira la regulación del procedimiento de formación de estos pactos. El origen de esta duda de la doctrina científica se halla en el art. 34. 5 TUEL que dice: «Oye l'accordo comporti variazione degli strumenti urbanistici, l'adesione del sindaco allo stesso deve essere ratificata dal consiglio comunale entro trenta giorni a pena di decadenza». Este precepto se puede interpretar en el sentido de que incumbe al Alcalde, y no al Consejo, como correspondería de conformidad con la ordinaria distribución de competencias fijada por el TUEL, decidir sobre la adhesión del Ayuntamiento a los acuerdos de programa. Un sector de la doctrina, mayoritario en la actualidad, defiende que no existen elementos en esta norma que autoricen a sostener que, a través de la regulación de los acuerdos de programa, el Legislador haya querido introducir derogaciones al ordinario reparto de las competencias entre los órganos de las Administraciones contrayentes (vid., p. ej., G. GRECO «Accordi di programma e procedimento amministrativo», op. cit., p. 54; G. MANFREDI, «Modelli contrattuali dell'azione amministrativa: l'acoordo di programma», op. cit., p. 370; G. SANTOMAURO «L'accordo di programma: procedimento di formazione ed approvazione -natura giuridica- effetti ed esecuzione», op. cit., p. 1331). En este sentido se ha pronunciado también el Consiglio di Stato (vid. S. Cons. Stato, Sez. VI, de 7 de febrero de 1996, n. ${ }^{\circ} 182$; S. Cons. Stato, Sez. VI, de 14 de julio de 2000, n. $\left.{ }^{\circ} 25\right)$. Frente a esta posición, otro sector de la doctrina, minoritario, entiende, realizando una interpretación a contrario del citado art. 34 TUEL, que dicho precepto atribuye directamente al órgano unipersonal el poder, no sólo de convocar la conferencia, sino también la potestad de expresar la voluntad de la respectiva Administración, y, por tanto, la capacidad de vincularla respecto de lo pactado en el acuerdo de programa, salvo cuando, como prevé el apartado 5 de dicho artículo, éste conlleve una variación de los planes urbanísticos vigentes, en cuyo caso será necesaria la sucesiva ratificación por el Consejo municipa (cfr. E. MAGGIORA Il nuovo ordinamento delle outonomie locali, Firenze, Noccioli, 2000, pp. 137-138; V. BALDINI, «L'accordo di programma nella legislazione regionale», op. cit, pp. 16481649 , nota 101; y, con reservas, F. MANGANARO, «Rilievi sugli accordi tra pubbliche amministrazioni», op. cit., p. 381).

55 Cfr. C. Proti, «Commento all'art. 27», L'autonomia locale, a cargo de L. PaPiano, Pragma, Bologna, 1991, p. 298; G. MANFREDI, «Modelli contranuali dell'azione amministrativa: l'accordo di programa», op. cit., pp. 370-371.

${ }^{56}$ Cfr. G. PeRULLI, «L'iter preliminare all'accordo di programa», Riv. trim. appalti, n. ${ }^{\circ} 4,1992$, p. 734; G. MANFREDI, Accordi e azione amministrativa, Giappichelli, Torino, 2001, p. 67.
} 
necesaria una plena identificación entre las Administraciones convocadas a la conferencia y aquellas que finalmente se adhieren a los acuerdos, ya que estos investigadores configuran como decisivo el momento terminal del procedimiento y el consenso alcanzado en dicha sede, es decir, consideran que la norma se refiere al consenso de todas las Administraciones interesadas en la suscripción del texto definitivo de los acuerdos de programa $^{57}$. Esta segunda interpretación presenta, frente a la anterior, la ventaja de que elimina el riesgo de permanente parálisis de este instrumento jurídico ${ }^{58}$.

Finalmente, el consenso de las Administraciones públicas interesadas, en el sentido que se acaba de precisar, elaborado y expresado por los órganos competentes, se testimonia mediante la firma por los representantes legales de las mismas de un documento en el que se contiene el acuerdo de $\operatorname{programa}^{59}$.

${ }^{57}$ En este sentido, Gianfranco Perulli precisa: «La soluzione del problema esegético [...] é che non è richiesta l'unanimità dei consensi dei soggetti chiamati all'aecordo di programma, quanto una adesione iniziale che dovrà poi trovare la conferma definitiva da parte delle amministrazioni interessate all'accordo finale. Ecco perché la norma ha fatto preciso richiamo alle amministrazioni interessate [...e non alle ammiministrazioni invitate o a quelle partecipanti...], laddove non viene richiesto un consenso unanime inteso in senso statico ed assoluto, ma un consenso qualificabile come consenso delle volontà; dinamico e riferito esclusivamente alle ammininistrazioni interessate [...a quelle che hanno concretamente dimostrato interesse all'accordo...]. E il consenso delle amministrazione interessate all'accordo (meglio al programma), vale a dire di quei soggetti che dopo le varie tappe della procedura hanno finito per dare la propria adesione conclusiva. Altre si sono perse per strada, hanno rinunciato, non si sono sentite in grado di aderire all'iniziativa. In ques'ottica il consenso unanime non è presupposto ma è concetto finale, strettamente connesso alla fase terminale tipica della formalizzazion dell'accordo» («L'iter preliminare all'accordo di programma», op. cit, 1992. p. 738).

${ }^{58}$ Cfr. S. Cons. Stato, Sez. VI, de 7 de febrero de 1996, n. ${ }^{\circ}$ 182. En esta sentencia, el Consiglio di Stato afirma que la falta de participación de alguna de las Administraciones interesadas en un acuerdo de programa no comporta la invalidez del acto, sino la eficacia del mismo limitada a los sujetos que han participado.

59 Si bien es cierto que algún autor ha sostenido que es suficiente «[...] il concorso di singole e separate manifestazioni di volontà su di un unico testo [...]» (G. CoRso, «Gli aceordi di programma», op. cit., p. 9). Por otra parte, conviene, quizás, recordar que a los acuerdos de programa, en cuanto acuerdos interadministrativos, se les aplica el art. 11.2, al que remite al art. 15.2 de la L. n. ${ }^{\circ}$ 241/1990, 7 agosto, Nuove norme in materia di procedimento amministrativo e di dirino di accesso ai doctimenti amministrativi, que establece: "Gli accordi di cui al presente articolo devono essere stipulati, a pena di nullità, per atto scritto, salvo che la legge disponga altrimenti». Hay destacar que la L. r. Toscana n..$^{\circ}$ 76/1996, de 3 de septiembre, Disciplina degli accordi di programma e delle conferene dei sevizi, en su art. 9, precisa mucho más este aspecto de la firma de los acuerdos de programa al disponer: «2. L'accordo di programma, è firmato dai legali rappresentanti delle amministraziont ed enti pubiblici partecipanti. 3. Nel caso in cui l'accordo di programma produca l'effetto di variazioni de uno o piò atti di programmazione o pianificazione territoriale, i legali rappresentanti delle amministrazioni ed enti pubblici partecipanti devono firmare anche gli allegati cartografici eventualmetne necessari per legge». 


\section{C) La fase de exteriorización}

Una vez suscrito el acuerdo de programa llega el momento de que éste, conforme a lo dispuesto en el art. 34 TUEL, sea, en primer lugar, aprobado y, posteriormente, publicado.

\section{a) La aprobación de los acuerdos de programa}

De acuerdo con el artículo 34.4 TUEL, la aprobación de los acuerdos de programa se produce mediante un acto formal que puede asumir la forma del decreto del Presidente de la Región, del Presidente de la Provincia o del Alcalde. Como se puede observar, el artículo 34.4 TUEL se limita a atribuir el poder de aprobación a los sujetos que antes ha mencionado como titulares del poder de promoción, si bien no aplica expresamente para individuar en cada caso al concreto sujeto titular del poder de aprobación el criterio de la competencia primaria o prevalente, como sí hace para determinar cuál es el sujeto titular del poder de promoción. Esto ha llevado a algún autor a afirmar que la norma en cuestión, indirectamente, consiente la posibilidad de que la elección del órgano aprobante pueda ser efectuada en el propio acuerdo de programa, aun en disconformidad con el criterio de la competencia primaria o prevalente ${ }^{60}$. Sin embargo, empleando un criterio sistemático para interpretar esta disposición legal, parece que debe considerarse atribuida la competencia de aprobaci6n al mismo sujeto que ha actuado el poder de promoción, es decir, a la Administración que ostenta la competencia primaria o prevalente sobre el objeto del acuerdo de programa ${ }^{61}$.

\section{b) La publicación de los acuerdos de programa}

El art. 34.4 TUEL establece que los acuerdos de programa serán publicados en el Boletín Oficial de la Región. De este modo, el TUEL ha escogido el nivel regional como el ámbito de publicación de dichos acuerdos, con independencia de los Entes que participen en ellos. Hay que tener en cuenta que tanto los Estatutos de los entes territoriales, como los propios

\footnotetext{
${ }^{60}$ Vid. N. Olivetti RAson, «Vecchio e nuoyo per Roma Capitale», Riv. giur. urb., n. ${ }^{\circ}$ 2-3, 1991, p. 499.

${ }^{61}$ Cfr. E. Sticchi Damiani, Attività amministrativa consensuale e accordi di programma, op. cit., p. 182. En este sentido se suele pronunciar la jurisprudencia, vid., p. ej., la S. TAR Toscana, Sez. I, de 10 de junio de 1998, n. $^{\circ} 346$.
} 
acuerdos de programa pueden establecer formas complementarias de publicidad, como, por ejemplo, la publicación en determinados periódicos o tablones de anuncios ${ }^{62}$.

\section{2) La eficacia de los acuerdos de programa}

Tras la aprobación, entre las partes, o bien, tras la publicación, frente a terceros $^{63}$, los acuerdos de programa comienzan a desplegar sus efectos. Dentro de dichos efectos hay que distinguir necesariamente dos grupos: uno constituido por los efectos típicos que resultan de todo acuerdo de voluntades, y otro integrado por los efectos particulares que la Ley anuda a la firma de este particular tipo de pactos.

Respecto del primer grupo, hay que decir que el efecto inmediato de la perfección de un acuerdo de programa válido, como sucede con cualquier contrato, consiste en instaurar una reglamentación de la conducta de las partes, con el deber de su observancia. Por lo que se refiere al segundo grupo, los efectos particulares, el art. 34 TUEL liga a la celebración de determinados acuerdos de programa dos tipos de efectos. Por una parte, según el art. 34.4 TUEL, cuando el acuerdo es aprobado a través de un decreto del Presidente de la Región, éste puede producir los efectos en materia de ordenación urbana del acuerdo («intesa») regulado en el art. 81 del DPR n. ${ }^{\circ} 616 / 1977$, de 24 de julio ${ }^{64}$; por otra parte, según el art. 34.6,

\footnotetext{
${ }^{62}$ Según Carlo ModicA, tras su publicación, los acuerdos de programa entran en vigor de acuerdo con las reglas ordinarias de la vacatio legis («L'accordo di programma: istituzionalizzazione dell' $\mathrm{i}-$ nefficienza?», op. cit., p. 29).

${ }^{63}$ En este sentido se ha pronunciado, p. ej., el TAR Calabria, Sede di Cantazaro, Sez. I, en su S. de 14 de julio de 2000, n. ${ }^{\circ} 1121$, donde afirma: «[...] la necessità di ricollegare la decorrenza del termine per l'impugnazione all'adozione delle misure di pubblicità previste per l'atto che segna il culmine del procedimento ed a cui si ricollega l'operatività dell'accordo di programma, costituito senza dubbio dal decreto di approvazione dell'accordo di programma, di competenza del presidente della regione [...] Ritiene il Tribunale che, soprattutto laddove, come nel caso di specie, la variante interessi aree di una certa entità e non singoli appezzamenti, il termine per l'impugnazione dell'accordo e delle previsioni in esso contenute non possa che decorrere dalla pubblicazione del decreto del presidente della regione, alla cui adozione, come si è detto, è legata l'operatività dell'accordo di programma, in quanto essa è l'unica misura di pubblicità prevista per tale atto».
}

${ }^{64} \mathrm{El}$ tenor literal del art. 34.4 TUEL dice: «[...] L'accordo, qualora adottato con decreto del presidente della regione, produce gli effetti dell'intesa di cui all'articolo 81 del decreto del Presidente della Repubblica 24 luglio 1977, n. ${ }^{\circ} 616$, determinando le eventuali e conseguenti variazioni degli strumenti urbanistici e sostituendo le concessioni edilizie, sempre che vi sia l'assenso del comune interessato». Los efectos urbanísticos consisten fundamentalmente en la posibilidad de introducir variaciones en los instrumentos de planeamiento y en la posibilidad de sustituir licencias, siempre que exista la conformidad del Ayuntamiento interesado. A propósito de esta capacidad de los acuerdos de programa para modificar instrumentos urbanísticos, hay que precisar que la jurisprudencia- 
la aprobación del acuerdo de programa comporta la declaración de utilidad pública, indiferibilidad y urgencia de ciertas obras públicas, si bien esta declaración deja de ser eficaz si dichas obras no se inician en un plazo de tres años ${ }^{65}$.

\section{3) La ejecución de los acuerdos de programa y su control}

Tras la aprobación y publicación de un acuerdo de programa debe procederse a la realización de las actividades previstas en él. Las Administraciones participantes en dicho acuerdo pueden optar por distintos sistemas para llevar a cabo la gestión de la ejecución de las obligaciones contenidas en el mismo. En efecto, pueden establecer un comité de gestión, crear un ente al cual confiar tal tarea, o bien delegar dicha gestión en una de las Administraciones participantes.

Sobre esta ejecución de lo contenido en los acuerdos de programa, el TUEL establece un sistema de control, que incluye la ejecución coactiva de las eventuales disposiciones violadas de dichos acuerdos, con el fin de garantizar la correcta actuación del proyecto para cuya realización cada acuerdo de programa ha sido estipulado. Conforme a lo dispuesto en el art. 34.7 TUEL esta vigilancia sobre la ejecución de los acuerdos de programa se confía a un colegio presidido por el Presidente de la Región, o de la Provincia o por el Alcalde ${ }^{66}$, y compuesto por representantes de las Administraciones que han suscrito el acuerdo de programa, así como por el Comi-

ha afirmado recientemente que tal capacidad no se limita a los instrumentos de ámbito municipal, sino también supramunicipal. En este sentido en la S. Cons. Stato, Sez. VI, de 14 de julio de 2000, n. ${ }^{\circ}$ 25/2001, se afirma: «La realizzazione di opere ed interventi che comportino la modifica di un piano territoriale paesistico può rientrare nel contenuto di un accordo di programa, non potendosi ritenere che l'art. 27 commi 4 e 5 della legge 142/1990 limiti l'oggetto dell'accordo di programma alla variaziane dei soli strumenti urbanistici. Lo strumento dell'accordo di programma, infatti, ha ormai portata generale ed i suoi limiti oggettivi devono essere individuati con il solo riferimento all'ampia definizione, contenuta nel comma 1 dell'art. 27 della legge 142/1990».

${ }^{65}$ Literalmente este precepto dice: «6. Per l'approvazione di progetti di opere pubbliche comprese nei programmi dell'amministrazione e per le quali siano immediatamente utilizzabili i relativi finanziamenti si procede a norma dei precedenti commi. L'approvazione dell'accordo di programma comporta la dichiarazione di pubblica utilità indifferibilità ed urgenza delle medesime opere; tale dichiarazione cessa di avere efficacia se le opere non hanno avuto inizio entro tre anni».

${ }^{66}$ La aplicación del criterio sistemático para interpretar este art. 34, ya empleado para atribuir al ente promotor la presidencia de la conferencia inicial, lleva a atribuir a este mismo ente la presidencia del colegio de vigilancia. En este sentido, el art. 12 apartado 2 de la L. r. Toscana n. ${ }^{\circ}$ 76/1996, de 3 de septiembre, Disciplina degli accordi di programma e delle conferenze di servizi, precisa: «Il collegio [...] è presieduto dal legale rappresentante dell'amministrazione che approva con propio atto l'accordo di programma. 
sario del Gobierno en la Región o por el Prefecto en la Provincia, si en el acuerdo participan Administraciones estatales o Entes públicos nacionales ${ }^{67}$. El art 34.8 TUEL establece que, en el caso de que intervengan dos o más Regiones limítrofes, el colegio de vigilancia será presidido por un representante de la Presidencia del Consejo de Ministros, y estará compuesto por representantes de todas las Regiones que hayan participado en el acuerdo. Respecto del funcionamiento de este colegio, un sector de la doctrina sostiene que se trata de un «collegio perfetto», y que por lo tanto debe adoptar las decisiones por unanimidad, en coherencia con el principio consensual que informa el proceso de celebración de los acuerdos de progra$\mathrm{ma}^{68}$. Por el contrario, otro sector doctrinal estima que este colegio debe deliberar según el principio mayoritario, salvo que se establezca otra cosa ${ }^{69}$. Esta segunda interpretación parece la más razonable, ya que si el colegio de vigilancia tuviese que actuar de acuerdo con la regla de la unanimidad no tendría sentido otorgar, como prevé el art. 34 TUEL, poderes de sustitución a este colegio, pues bastaría tan sólo el voto de uno de sus miembros, el representante de la Administración incumplidora, para que tales poderes se conviertan en papel mojado ${ }^{70}$. Fundamentalmente, las Administraciones públicas al suscribir los acuerdos de programa suelen atribuir a este colegio de vigilancia dos tipos de labores: por lado, funciones de vigilancia en sentido estricto, y, por otro lado, funciones compositivas ${ }^{71}$. Respecto de las pri-

${ }^{67}$ Estos Entes públicos no pueden participar directamente mediante representantes propios en el colegio de vigilancia. La tutela de sus intereses corresponde, bien Comisario del Gobierno o bien al Prefecto, pero el texto del artículo 34 no especifica cuando tal tutela le corresponde cada uno de estos sujetos. La Circular del Ministerio del Interior n. ${ }^{\circ}$ 17110/1271/1, de 7 de junio de 1990, establece que el Comisario del Gobierno debe intervenir cuando el poder de promoción del acuerdo corresponda a la Región, mientras que el Prefecto debe participar en el colegio de vigilancia cuando dicho poder corresponda al Ayuntamiento o a la Provincia (vid. M.A. VENCHI CARnEVALE, «I servizi pubblici e le forme associative e di cooperazione», Riv. amm., n. ${ }^{\circ} 142$, fasc. 4, 1991, p. 500).

${ }^{68}$ Cfr. G. MANFREDI, «Modelli contranuali dell'azione amministrativa: 1'accordo di programa», op. cit, p. 376, A. CIMEllaro y A. FerRuti, La conferenza di servizi e l'accordo di programma, op. cit., pp. $150-151$.

69 Cfr. C. ModicA, «L'accordo di programma: istitutonalizzazione dell'inefficienza?», op. cit., p. 29. Por el principio mayoritario se inclina también Salvador Nocera (Gli accordi di programma per l'integrazione scolastica e sociale delle persone con handicap, Unicopli, Milano, 1994, p. 79). En este sentido, la L. r. Toscana n. ${ }^{\circ}$ 76/1996, de 3 de septiembre, Disciplina degli accordi di programma e delle conferenze di servizi, en su art. 12 apartado 2 dispone: «[...] Il collegio delibera a maggioranza dei componenti $[\ldots] »$.

70 Por el resto, y como señala Salvador NocERA, dado que se trata de un órgano colegial, valen para el todas la normas sobre la constitución de los órganos colegiados y sobre el funcionamiento de los mismos (Gli accordi di programma per l'integraizione scolastica e sociale delle persone con handicap, op. cit., p. 79).

71 Aunque no es infrecuente que se le asignen otras funciones como: individuar los obstáculos de hecho y de derecho que se interponen a la ejecución del acuerdo de programa proponiendo solucio- 
meras, hay que destacar que la actividad principal del colegio es vigilar el puntual cumplimiento del acuerdo. En caso de que se produzca un retraso en la ejecución o un incumplimiento de lo dispuesto en el acuerdo de programa, el colegio de vigilancia intima al sujeto responsable de dicha demora o incumplimiento, dándole un plazo para proceder a realizar cuanto le ha sido requerido. Dicho plazo ha de ser congruente con los objetivos generales y los plazos fijados en el acuerdo ${ }^{72}$. En el caso de que el sujeto intimado no corrija su actitud, y cumpla lo pactado, en los acuerdos de programa suele preverse que el colegio nombre a un comisario ad acta para que adopte los actos en los cuales se ha verificado la inercia o el retraso, o bien se dispone que sea el propio colegio quien dicte tales actos ${ }^{73}$. Generalmente, se establece que los gastos derivados del ejercicio de esta facultad de subrogación son a cargo de la Administración incumplidora, precisándose que tales gastos han de ser determinados, siempre que sea posible, de acuerdo con las tarifas profesionales ofíciales. En segundo lugar, habitualmente, atribuye a este colegio de vigilancia una función compositiva. Esto significa que en el acuerdo de programa se establece que toda controversia derivada de la ejecución del acuerdo puede ser definida en vía amigable por el colegio de vigilancia. Asimismo; es frecuente encontrar la previsión de que, en caso de que no sea posible, dicha cuestión será devuelta al órgano competente. Para el desarrollo de estas actividades el colegio de vigilancia puede requerir documentos e informaciones a las Administraciones participantes, convocar funcionarios y representantes y llevar a cabo inspecciones ${ }^{74}$. Para ayudarle en sus labores de vigilancia sobre la ejecución de lo establecido en el acuerdo de programa, el colegio puede valerse de una secretaría técnica. A la cabeza de ésta, como norma general, se halla el funcionario responsable de la ejecución del acuerdo, que actúa como secretario del colegio. Este funcionario, que normalmente es elegido entre los diri-

nes idóneas para su remoción; proponer la prorroga del acuerdo; aprobar eventuales modificaciones del acuerdo de programa. Hay que subrayar que la atribución de funciones al colegio de vigilancia ha de hacerse teniendo en cuenta que la naturaleza de este colegio es la de un órgano de vigilancia y control, cosa que impide que se le encomiende cualquier actividad de ejecución de lo dispuesto en el acuerdo de programa. Este tipo de actividades deben encomendársele al órgano u ente encargado de la gestión de la ejecución de cada acuerdo de programa.

72 En ocasiones, los acuerdos establecen que el plazo o bien no puede ser inferior o bien no puede ser superior a un determinado número de días.

${ }^{73}$ Además, en estos supuestos, corresponde al colegio de vigilancia aplicar las sanciones que hayan podido ser previstas en el acuerdo de programa.

${ }^{74}$ Hay que señalar que, en la práctica, se suele reconocer al colegio de vigilancia el derecho a acceder a todos los documentos relativos al acuerdo de programa en posesión de los sujetos firmantes, quienes se comprometen, además, a proporcionarle cualquier información directamente relacionada con la ejecución del acuerdo de programa en cuestión. 
gentes y funcionarios de la Administración que aprueba el acuerdo de programa, se encarga de levantar acta de las sesiones, señala al colegio el estado de ejecuci6n del acuerdo, mantiene los contactos necesarios con las oficinas y estructuras técnicas de las Administraciones participantes, y, en definitiva, realiza cualquier actividad útil al fin de coordinar las acciones de los Entes participantes en el acuerdo. Además, en los acuerdos de programa se suele especificar que corresponde a este funcionario presentar al colegio de vigilancia un informe sobre el estado de cumplimiento de las obligaciones asumidas por cada una de las Administraciones participantes.

\section{BIBLIOGRAFÍA CITADA}

BALDINI, Vincenzo, «L'accordo di programma nella legislazione regionale», Regioni, n. ${ }^{\circ}$ 6, 1992, pp. 1617 ss.

BAssi, Nicola, «Commento all'articolo 34», Testo Unico degli Enti Locali, coordinado por Vittorio Italia, vol. I, tomo I, Giuffrè, Milano, 2000, pp. 396 ss.

BRETINI, Pierluigi, «La conferenza di servizi», Dir amm., n. ${ }^{\circ}$ 2, 1997, pp. 325 ss.

CAIAFFA, Fabio, «Le nuove frontiere dell'attività amministrativa consensuale: gli accordi di programma», Nuova rass., n. ${ }^{\circ} 3-4,1993$, pp. 233 ss.

CARavita, Beniamino, «Gli accordi di programma», Azienditalia, n. ${ }^{\circ} 9$, 1990, pp. 38 ss.

CARTEI, Gian Franco, «Gli accordi di programma nel diritto comunitario e nazionale», Riv. it. dir pubbl. Com., n. ${ }^{\circ} 1,1991$, pp. 49 ss.

CHIEFFI, Lorenzo, «Gli strumenti per il coordinamento della ricerca scientifica: l'accordo di programma introdotto dalla legge n. 168 del 1989», Riv. giur mezz., n. ${ }^{\circ}$ 3, 1992, pp. 713 ss.

Cimellaro, Antonino; Ferruti, Andrea, La conferenza di servizi e l'accordo di programma, $2^{\mathrm{a}}$ ed., Maggioli, Rimini, 2000.

Civitarese Matteucci, Stefano, «Accordo di programma», Enc. Dir., Giuffrè, Milano, 1999, pp. 9 ss.

COMPORTI, Gian Domenico, Il coordinamento infrastrutturale. Tecniche e garanzie, Giuffrè, Milano, 1996. 
Conso, Guido, «Gli accordi di programa» Riv. giur. mezz., n. ${ }^{\circ}$ 1, 1988, pp.5 ss.

- «Gli accordi di programma», L'accordo nell'azione amministrativa, a cargo de Alfonso Masucci, FORMEZ, Roma, 1988, pp. 51 ss.

Di GASPARE, Giuseppe, «L'accordo di programma: strutture, efficacía giuridica e problemi di gestioni», Regioni, n. $^{\circ} 2,1988$, pp. 278 ss.

Di MARCO, Carlo, «I rapporti Stato-Regioni-Enti locali nel sistema dell'amministrazione pattizia», Regioni, n. ${ }^{\circ} 3,1999$, pp. 471 ss.

D'ORTA, Carlo, «I programmi integrati mediterranei in Italia: disciplina e prima attuazione», Riv. giur mezz., n. ${ }^{\circ} 1,1988$, pp. 101 ss.

FALCON, Giandomenico, «Gli aecordi tra amministrazioni e tra amministrazioni e privato», La semplificazione amministrativa, a cargo de Luciano Vandelli y Gianluca Gardini, Maggioli, Rimini, 1999, pp. 149 ss.

Ferrara, Rosario, Gli accordi di programma. Potere, poteri pubblici e modelli dell'amministrazione concertata, Cedam, Padova, 1993.

FIORITTO, Alfredo, «Accordi di programma e contrats plan: somiglianze e divergenze», Riv. giur. mezz, n. ${ }^{\circ} 3,1989$, pp. 867 ss.

Giannini, Massimo Severo, Diritto pubblico dell'economia, Il Mulino, Bologna, 1993.

GRECO, Guido, «Commento all'art. 27», Le autonomie locali, coordinado por Vittorio Italia y Mario Bassani, tomo I, Giuffrè, Milano, 1990, pp. 379 ss.

- «Accordi di programma e procedimento amministrativo», Dir. eco., $\mathrm{n}$. 2, 1991, pp. 335 ss.

- «Accordi di programma e procedimento amministrativo», I rapporti fra cittadini e istituzioni nelle recenti leggi di riforma delle autonomie locali e del procedimento amministrativo. Atti del Convegno di Milano, 14 gennaio 1991, Giuffrè, Milano, 1992, pp. 47 ss.

GuALANDI, Federico, «L'accordo di programma nella disciplina urbanistica», Riv. giur. edil., fasc. 1, 1996, pp. 27 ss. 
MAGGIORA, Enrico, Il nuovo ordinamento delle autonomie locali, Noccioli, Firenze, 2000.

MANFREDI, Giuseppe, «Modelli contrattuali dell'azione amministrativa: l'accordo di programma», Regioni, n. ${ }^{\circ} 2$, 1992, pp. 349 ss.

— «Commento all'art. 15 L. 7 agosto 1990 n. 241», Leggi civ. comment, n. $^{\circ} 1,1995$, pp. 87 ss.

- Accordi e azione amministrativa, Giappichelli, Torino, 2001.

MANGARO, Francesco, «Rilievi sugli accordi tra pubbliche amministrazioni», Scritti per Enzo Silvestri, Giuffrè, Milano, 1992, pp. 337 ss.

- «Il coordinarnento degli interessi pubblici negli accordí di programma», Giur. it., n. ${ }^{\circ} 2,1994$, pp. 36 ss.

ModicA, Carlo, «L'accordo di programma: istituzionalizzazione dell'inefficienza?», Riv. giur. mezz., n. ${ }^{\circ} 1,1991$, pp. 5 ss.

Nocera, Salvatore, Gli accordi di programma per l'integrazione scolastica e sociale delle persone con handicap. Dalla legge n. 142/1990 sulle autonomie locali alla legge-quadro n. 104/1992, Unicopli, Milano, 1994.

Olivetti Rason, Nino, «Vecchio e nuovo per Roma Capitale», Riv. giur. urb., n. ${ }^{\circ} 2-3,1991$, pp. 499 ss.

Pallottino, Michele, «Strumenti giuridici di concertazione amministrativa: L'accordo di programa», Riv. amm., fasc. 12, 1983, pp. 847 ss.

PAOLuCCI, Francesco, «I soggetti degli accordi di programma», Livelli e contenuti della pianificazione territoriale, a cargo de Emilio FERRARI, Nazareno SAITTA y Aldo Tigano, Giuffrè Midano, 2001, pp. 297 ss.

PERICU, Giuseppe, «L'attività consensuale della amministrazione pubblica», Diritto amministrativo, a cargo de Leopoldo MAZZAROLLi y otros, tomo II, $3^{\text {a }}$ ed., Monduzzi Editore, Bologna, 2001, pp. 1611 ss.

PERULLI, Gianfranco, «L'iter preliminare all'accordo di programma», Riv. trim. appalti, n. ${ }^{\circ}$, 1992, pp. 729 ss.

Piacentini, Pier Maria, «La conferenza di servizi: considerazioni generali», Riv. trim. appalti, n. ${ }^{\circ} 4,1989$, pp. 1131 ss. 
Predieri, Alberto, «Gli accordi di programma», Quad., reg., n. ${ }^{\circ}$ 4, 1991, pp. 957 ss.

PRESIDENZA DEL CONSIGLIO DEI MINISTRI-DiPARTIMENTO DELLA FUNZIONE PUBLICA, «Gli accordi di programma», La semplificazione dei procedimenti di spesa per gli investimenti pubblici, Istituto Poligrafico e Zecca dello Stato, Roma, 1994, pp. 59 ss.

Pronti, Carlo, «Commento all'art. 27», L'autonomia locale, a cargo de Luigi Papiano, Pragma, Bologna, 1991, pp. 295 ss.

QuAGLiA, Mario Alberto, «La conferenza di servizi: profili procedimentali», Quad reg., n. ${ }^{\circ} 1-2,1991$, pp. 105 ss.

Samuelson, Paul A.; Nordhaus, William D., Economía, 14a ed., MacGraw-Hill, Madrid, 1994.

SANTOMAURO, Giovanni, «L'accordo di programma: procedimento di formazione ed approvazione -natura giuridica- effetti ed esecuzione», Com. it., n. ${ }^{\circ}$ 9, 1992, pp. 1327 ss.

Sciullo, Girolamo, «Sintonie e dissonanze fra le L. 8 giugno 1990 n. 142 e 7 agosto 1990 n. ${ }^{\circ}$ 241: riflessi sull'autonomia locale», Foro amm., 1990, pp. 2198 ss.

Sticchi Damiani, Ernesto, Attività amministrativa consensuale e accordi di programma, Giuffrè, Milano, 1992.

- «Gli accordi amministrativi», La contrattualizzazione dell'azione amministrativa, a cargo de C. Amirante, Giappichelli, Torino, 1993, pp. 23 ss.

TOMEI, Roberto, «Strumenti di coordinamento amministrativo per lo sviluppo del Mezzogiorno: 1'accordo di programma», Trib. amm. reg., n. ${ }^{\circ}$ 7-8, parte II, 1990, pp. 253 ss.

Travi, Aldo, «Le forme di cooperazione interlocale», Dir. amm., n. ${ }^{\circ} 4$, 1996, pp. 685 ss.

TURCo Liveri, Giuseppe, «Gli accordi di programma», Com. it., n. ${ }^{\circ}$ 9, 1995, pp. 1259 ss.

Venchi Carnevale, Maria Adelaide, «I servizi pubblici e le forme associative e di cooperazione», Riv. amm., n. ${ }^{\circ} 142$, fasc. 4, 1991, pp. 475 ss. 